\title{
THE STRUCTURE OF
}

\section{$n$-UNIFORM TRANSLATION HJELMSLEV PLANES}

\author{
BY
}

\author{
DAVID A. DRAKE
}

ABSTRACT. Affine or projective Hjelmslev planes are called 1-uniform (also strongly 1-uniform) if they are finite customary affine or projective planes. If $n>1$, an $n$-uniform affine or projective Hjelmslev plane is a (finite) Hjelmslev plane OU with the following property: for each point $P$ of $\mathscr{f l}$, the substructure $n-1 P$ of all neighbor points of $P$ is an $(n-1)$-uniform affine Hjelmslev plane. Associated with each point $P$ is a sequence of neighborhoods ${ }^{1} P \subset 2 P \subset \ldots$ $C^{n} P=\mathcal{U}$. For $i<n,{ }^{i} P$ is an $i$-uniform affine Hjelmslev plane under the induced incidence relation (for some parallel relation). Hjelmslev planes are called strongly $n$-uniform if they are $n$-uniform and possess one additional property; the additional property is designed to assure that the planes have epimorphic images which are strongly $(n-1)$-uniform. Henceforth, assume that $\mathscr{f}$ is a strongly $n$-uniform translation (affine) Hjelmslev plane. Let $\left({ }^{i} P\right)^{*}$ denote the incidence structure ${ }^{i} P$ together with the parallel relation induced therein by the parallel relation holding in $\mathcal{U}$. Then for all positive integers $i \leq n$ and all points $P$ and $Q$ of $\mathcal{Q},\left({ }^{i} P\right)^{*}$ and $\left({ }^{i} Q\right)^{*}$ are isomorphic strongly $i$-uniform translation Hjelmslev planes. Let ${ }^{i \mathscr{U}}$ denote this common $i$-uniform plane; $\left({ }^{i} \mathscr{U}\right)_{j}$, denote the "quotient" of "iथ modulo $i \mathscr{Q}$. The invariant $r=p^{x}$ of $\mathscr{Q}$ is the order of the ordinary translation plane $\left({ }^{n} \mathfrak{Q}\right)_{n-1}$. Then the translation group of $\mathfrak{U}$ is an abelian group with $2 x k$ cyclic summands, $k$ an integer $\leq n$; one calls $k$ the width of $\mathfrak{U}_{\text {. If }} 0 \leq j<i \leq n$, then $\left({ }^{i} \mathscr{Q}\right)_{j}$ is a strongly $(i-\bar{j})$-uniform translation Hjelmslev plane; if also $j \geq k,\left({ }^{i} \mathfrak{U}\right)_{j}$ and $\left({ }^{i-k}{ }^{2}\right)_{j-k}$ are isomorphic. Then if $\mathcal{U}(i)$ denotes $\left({ }^{i} \mathfrak{U}\right)_{i-1}, \mathcal{Q}(1), \ldots, \mathscr{U}^{j}(n)$ is a periodic sequence of ordinary translation planes (all of order $r$ ) whose period is divisible by $k$. It is proved that if $T_{1}, \cdots, T_{k}$ is an arbitrary sequence of translation planes with common order and if $n \geq k$, then there exists a strongly $n$-uniform translation Hjelmslev plane $\mathcal{U}$ of width $k$ such that $\mathscr{U}(i) \cong T_{i}$ for $i \leq k$. The proof of this result depends heavily upon a characterization of the class of strongly $n$-uniform translation Hjelmslev planes which is given in this paper. This characterization is given in terms of the constructibility of the $n$-uniform planes from the $(n-1)$-uniform planes by means of group congruences.

Introduction. Hjelmslev planes are generalizations of customary affine and projective planes in which distinct lines may intersect in more than a single point. Throughout this paper, we will refer to a Hjelmslev plane as an $H$-plane. One calls a finite $H$-plane 1 -uniform if it is a customary affine or projective $H$-plane:

Received by the editors May 14, 1971.

AMS (MOS) subject classifications (1970). Primary 05B25, 50D35; Secondary 05B30.

Key words and phrases. Translation, translation plane, group congruence, Hjelmslev plane, $n$-uniform, strongly $n$-uniform, height $n$, level $n$. 
For a finite $H$-plane (affine or projective) to be called $n$-uniform, $n>1$, one demands that all its maximal point neighborhoods be $(n-1)$-uniform affine $H$-planes. (There is a second requirement as well; see Definition 1.4.)

$S$ trongly $n$-uniform $H$-planes are defined to be $n$-uniform $H$-planes which satisfy a condition called Property S (see Definition 2.1). The assumption of Property $S$ in an $n$-uniform projective $H$-plane $\mathscr{U}$ is equivalent [4, Theorem 4.2] to assuming that $\mathscr{U}$ has a strongly $(n-1)$-uniform epimorphic image under a certain prescribed epimorphism. While we do not investigate the corresponding question for affine $H$-planes, at least we know that strongly $n$-uniform translation affine $H$ planes do have strongly $i$-uniform epimorphic images for $1 \leq i \leq n-1$ (see Proposition 2.9 for a somewhat stronger result).

In 1954, André [1] proved that all translation planes can be represented by group congruences. In 1962, Lüneburg [9] gave two methods for constructing 2uniform translation (affine) $\mathrm{H}$-planes from a given finite translation plane, i.e. from a given 1-uniform translation $H$-plane. Both methods involve the use of group congruences; both may be applied to any finite translation plane to yield 2-uniform translation $H$-planes. Lüneburg proved [9, Satz 9.1 and Satz 9.2] that the two constructions yield all 2 -uniform (affine) translation $H$-planes.

Henceforth, "translation $H$-plane" will be taken to mean translation affine $H$ plane. In $\$ 3$ of this paper, we describe two generalizations of Lüneburg's constructions. We prove (Theorem 4.1 (b)) that, if either of these is applied to a strongly $n$-uniform translation $H$-plane, the result is a strongly $(n+1)$-uniform translation $H$-plane. One of the two constructions may be applied to any strongly $n$-uniform translation $H$-plane; the other may be applied precisely to the ones which have elementary abelian translation groups. Conversely (Theorem 4.1 (a)), every strongly $(n+1)$-uniform translation $H$-plane can be constructed by one of the two methods from some strongly $n$-uniform translation $H$-plane. Since $n$-uniform implies strongly $n$-uniform when $n=1$ or 2 , the above results are all generalizations of the corresponding theorems of Lüneburg.

In [2], Artmann characterized the projective $H$-planes of "height $n, " n \geq 2$, as the class of projective $H$-planes obtained by applying a particular construction process to the projective $H$-planes of height $n-1$. Artmann's construction procedure differs fundamentally from the generalized Lüneburg constructions given in this paper in the following respect: in Artmann's construction, the H-plane of height $n-1$ is recaptured by taking an epimorphic image of the constructed $H$ plane; in the constructions presented in this paper, the $(n-1)$-uniform $H$-plane appears as a substructure of the constructed $n$-uniform $H$-plane.

In [6], the author proved that if $\mathcal{U}$ is an $n$-uniform translation $H$-plane whose associated customary affine plane has order $p^{x}, p$ a prime, then the (abelian) 
translation group of $\mathscr{U}$ contains $2 x k$ direct summands where $k$ is an integer $\leq n$. We call $k$ the width of $\mathcal{U}$. If $k=n$, we say $\mathcal{U}$ is borizontal; if $k=1$, we say $\mathcal{U}$ is vertical. As a corollary to Theorem 4.1(b), we obtain the result that all vertical $n$-uniform translation $H$-planes are strongly $n$-uniform.

Associated with each point $P$ of an $n$-uniform $H$-plane $\mathcal{U}$ is a sequence of neighborhoods: ${ }^{1} P,{ }^{2} P, \ldots,{ }^{n} P=\mathscr{U}$. For each $i<n$ and each point $P$, there exists a parallel relation on the lines of ${ }^{i} P$ which makes ${ }^{i} P$ into an $i$-uniform affine $H$-plane under the incidence relation induced by $\mathscr{U}$ (Proposition 1.3). If $\mathcal{U}$ is strongly $n$-uniform, then ${ }^{i} P$ is strongly $i$-uniform (Proposition 2.2). If $\mathcal{U}$ is either an $n$-uniform translation $H$-plane or an affine $H$-plane belonging to a strongly $n$-uniform projective $H$-plane, then ${ }^{i} P$ may be given the parallel relation induced by the parallel relation holding in $\mathcal{U}$ (Propositions 1.4 and 2.3$)$. Let $\left({ }^{i} P\right)^{*}$ denote ${ }^{i} P$ together with the induced parallel relation. If $\mathcal{U}$ is a translation $H$-plane, so is $\left({ }^{i} P\right)^{*}$; and $\left({ }^{i} P\right)^{*}$ and $\left({ }^{i} Q\right)^{*}$ are isomorphic for all points $P$ and $Q$ of $\mathcal{U}$ (Proposition 1.4).

If $\mathscr{U}$ is an $n$-uniform translation $H$-plane, we define $\left({ }^{i} \mathscr{U}\right)_{j}$ to be the "quotient" of $\left({ }^{i} P\right)^{*}$ modulo $\left({ }^{j} P\right)^{*}$ for $0 \leq j<i \leq n$. We prove (Proposition 1.10) that $\left({ }^{i} \mathfrak{U}\right)_{j} \cong$ $\left({ }^{i-k} \mathfrak{U}\right)_{j-k}$ for $n \geq i>j \geq k$ where $\bar{k}$ is the width of $\mathcal{Q}$. Let $\mathscr{U}(i)$ denote $\left({ }^{i} \mathfrak{U}\right)_{i-1}$. Then the sequence of ordinary translation planes $\mathscr{U}(1), \ldots, \mathfrak{U}(n)$ clearly has period divisible by $k$. If $\mathscr{U}$ is a strongly $n$-uniform translation $H$-plane, then the $\left({ }^{i} \mathfrak{U}\right)_{j}$ are strongly $(i-j)$-uniform translation $H$-planes (Proposition 2.9).

The invariant of an $n$-uniform $H$-plane is defined to be the order of the associated customary affine or projective plane. If $\mathcal{U}$ is a strongly $n$-uniform translation $H$-plane with invariant $r$, then every $\left({ }^{i} \mathcal{U}\right)_{j}$ also has invariant $r$, and every $\mathscr{U}(i)$ has order $r$. The following theorem is the second major result obtained in this paper.

Theorem 6.3. Let $\mathbb{E}$ and $\mathbb{S}$ be borizontal translation H-planes with a common invariant $r$. Suppose that $\mathfrak{E}$ is strongly $i$-uniform and that $\mathfrak{S}$ is strongly j-uniform. Then there is a borizontal strongly $(i+j)$-uniform translation H-plane O with invariant $r$ such that $\left.\left({ }^{(} \mathfrak{U}\right)\right)_{0} \cong \mathbb{B}$ and $(i+j \mathfrak{Q})_{i} \cong \mathbb{E}$.

The proof of Theorem 6.3 is heavily dependent upon the constructive characterization of the class of strongly $n$-uniform translation $H$-planes given in Theorem 4.1. By an easy induction proof, Theorem 6.3 is generalized to Corollary 6.4 which states that strings of arbitrarily many $H$-planes of the specified type can be "pasted" together as "factors" of one large horizontal H-plane. Remark 6.5 (taken in conjunction with Remark 6.2 ) says that one can construct a strongly $n$ uniform translation $H$-plane $\mathcal{U}$ of width $k$ and invariant $r$ so that the first $k$ planes of the sequence $\mathscr{U}(1), \ldots, \mathcal{U}(n)$ are any ordinary translation planes of 
order $r$ taken in any order. Here $r$ is any prime power, and $k$ and $n$ are any positive integers satisfying $k \leq n$.

Since the definition of $n$-uniform H-planes includes the assumption of finiteness (see Definition 1.4), all results obtained in the paper refer only to finite $H$. planes.

1. $n$-uniform translation $H$-planes. Let $\|$ denote an equivalence relation defined on the line set of an incidence structure $(\$, \notin, \epsilon)$. Then $(\$, \&, \epsilon, \|)$ is called an incidence structure with parallelism, and $\|$ is called a parallelism defined on $(\$, \otimes, \epsilon)$. Let $\mathscr{U}$ and $\mathbb{Z}$ be incidence structures with parallelism. Then a bomomorphism from $\mathscr{U}$ to $\mathscr{Q}$ is an incidence-structure homomorphism $\phi$ from $\mathscr{U}$ to $\mathscr{W}$ such that $\phi(g) \| \phi(b)$ whenever $g \| b$. An isomorphism is an incidence-structure isomorphism which satisfies $\phi(g) \| \phi(b)$ if and only if $g \| b$. In an arbitrary incidence structure, one defines points $P$ and $Q$ to be neighbor and writes $P \sim Q$ to mean that $P$ and $Q$ have at least two common incident lines. Lines $g$ and $b$ are said to be (affinely) neighbor, and one writes $g \sim b$ to mean that each point incident with either of $g$ or $b$ possesses a neighbor point incident with the other of $g$ or $b$.

Definition 1.1. An incidence structure with parallelism, $\mathscr{U}=(\Re, \mathbb{E}, \epsilon, \|)$ is said to be an affine H-plane provided that the following four axioms are satisfied:

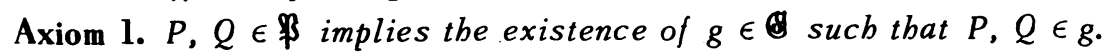

Axiom 2. If $|g \cap h| \geq 1$, then $|g \cap b| \geq 2$ if and only if $g \sim b$.

Axiom 3. There exists an epimorphism $\phi$ from a to an ordinary affine plane $\bar{\alpha}$ satisfying:

(a) $\phi(P)=\phi(Q) \Leftrightarrow P \sim Q$.

(b) $\phi(g)=\phi(b) \Leftrightarrow g \sim b$.

(c) $|g \cap b|=0 \Rightarrow \phi(g) \| \phi(b)$.

Axiom 4. Every point of $¥$ lies on exactly one line of each \|-class.

Definition 1.2 An incidence structure $\pi=(\Re, \notin, \epsilon)$ is called a projective $H$ plane provided that $\pi$ satisfies Axiom 1, the dual of Axiom 1, and the Axiom 5 stated below.

Axiom 5. There exists an epimorphism $\phi$ from $\pi$ to an ordinary projective plane $\bar{\pi}$ satisfying:

(a) $\phi(P)=\phi(Q) \Leftrightarrow P \sim Q$.

(b) $\phi(g)=\phi(b) \Leftrightarrow g \sim b$.

To interpret Axiom 5 (b) correctly one must know that one defines $g \sim b$ in a projective $H$-plane if and only if $|g \cap h|>\dot{1}$.

The above definition for projective $H$-planes together with another equivalent definition appear in Dembowski [3, pp. 291-293]. The above definition for affine $H$-planes is due to Lüneburg [9, Satz 2.6] (see also [9, Definition 2.3]). The reader is warned that Lüneburg's characterization of affine $H$-planes is misquoted by Dembowski [3, p. 296]. Dembowski's definition fails to include the parallelism 
as part of the structure of the affine $H$-plane and in addition weakens the axioms in two distinct ways. That Dembowski's definition is indeed more general than the one given above is demonstrated by an example due to Bacon [10]. While we use Lüneburg's definition for affine $H$-planes, we will often call an incidence structure $\mathfrak{U}=(\Re, \mathbb{B}, \epsilon)$ an affine $H$-plane or will say that "Ul is an affine $H$-plane under some parallelism" if there exists a parallelism \| on $\mathcal{A}$ such that $(\Re, \mathbb{Q},\|\|$,$) is an affine H$-plane.

Definition 1.3. Let $P$ be a point of an $H$-plane $\pi$. We define $\bar{P}$ to be the following incidence structure. The points of $\bar{P}$ are the points $Q$ of $\pi$ such that $Q \sim P$. The lines of $\bar{P}$ are the nonempty sets $b^{P}=b \cap \bar{P}, b$ being a line of $\pi$. Incidence is given by inclusion.

Definition 1.4. A 1-uniform affine (projective) $H$-plane is defined to be a finite ordinary affine (projective) plane. One calls a finite affine or projective $H$-plane $\pi n$-uniform $(n \geq 2)$ provided that:

(a) for each point $P$ in $\pi$, there exists a parallelism on $\bar{P}$ under which $\bar{P}$ becomes an $(n-1)$-uniform affine $H$-plane;

(b) for each $\bar{P}$, every line $b^{P}$ is the restriction of the same number of lines of $\pi$.

Associated with every affine or projective $H$-plane are two invariants denoted by $s$ and $t$. We may take $t$ to be the number of lines through a point $P$ which are neighbor to the line $g$ where $(P, g)$ is an arbitrary flag of the $H$-plane; then $s+t$ will denote the total number of lines incident with $P$. It is well known that $s / t$ is the order of the ordinary affine or projective plane associated with the $H$-plane (see [9] and [7]).

The reader should thoroughly acquaint himself with the content of the following proposition, as it will be used frequently throughout the paper, occasionally without reference. The result is proved in [4, Proposition 2.2]. To state the result, we need the following notation. Let $\pi$ be an $H$-plane with invariants $s, t$, and let $r$ denote $s / t$. Then we write "P $(\simeq i) Q$ " and read " $P$ is $i$-equivalent to $Q$ " to mean the point $P$ is joined to the point $Q$ by exactly $r^{i}$ lines; we write "P $(\sim i) Q$ " and read " $P$ is at least $i$-equivalent to $Q$ " to mean $P$ is joined to $Q$ by $r^{i}$ or more lines.

Proposition 1.1. Let $\pi$ be an n-uniform projective or affine H-plane. Then $\pi$ satisfies the following properties:

(1) If $r=s / t$, then $s=r^{n}$.

(2) If $P$ and $Q$ are distinct points of $\pi, P\left(\simeq_{i}\right) Q$ for some nonnegative integer $i<n$.

(3) The dual of (2) bolds in $\pi$ for lines with nonempty intersection.

(4) If $P \in b,|\{Q \in b: Q(\sim i) P\}|=r^{n-i}$ for $i=1,2, \cdots, n$.

(5) The dual of (4) bolds in $\pi$. 
(6) $(\simeq i)$ is an equivalence relation on the points of $\pi$ for $i=0,1, \ldots, n$.

(7) The following conditions imply $|h \cap k|>1: R, Q \in b ; R, S \in k ; R(\simeq i) Q$; $Q(\sim i+1) S ; i$ is a nonnegative integer $<n$.

(8) If $P$ is any point of $\pi$, the number of points $Q$ of $\pi$ such that $Q(\sim i) P$ is $r^{2(n-i)}$ for $i=1,2, \ldots, n$.

(9) Let $|b \cap k|=r^{i}$ with $i<n$. Then if $P \in b \cap k, b \cap k=\{Q \in b$ : $Q(\sim n-i) P\}$.

(10) The dual of (9) bolds in $\pi$.

(11) For each point $P$, each line of $\bar{P}$ is the restriction of exactly $r$ lines of $\pi$.

Condition (11) is to be found in the proof but not in the statement of [4, PropProposition 2.2.]

In light of Proposition 1.1 (1), an $n$-uniform $H$-plane may be thought of as having three invariants: $r, s$, and $t$. However, $s$ and $t$ are determined by $r$ and $n$; thus we shall write the invariant of an $n$-uniform $H$-plane $\pi$ to refer to $r$. Since $r=s / t$, the invariant of $\pi$ is the order of the ordinary affine or projective plane associated with $\pi$.

In order to state the next several results, we need the following notation. If $P$ is a point of an $n$-uniform (affine or projective) $H$-plane $\pi$, we write ${ }^{i} P$ to denote the incidence structure induced by $\pi$ in $\{Q: Q(\sim n-i) P\}, 0 \leq i \leq n$. If $i, j$ are integers such that $0 \leq i \leq j \leq n$, we write $\left[{ }^{i} P: j\right]$ to denote the incidence structure induced by ${ }^{j} P$ into the set of all points $Q$ of ${ }^{j} P$ which are joined to $P$ by at least $r^{j-i}$ lines of ${ }^{j} P$ (where $r$ is the invariant of $\pi$ ).

Lemma 1.2. Let $P$ be a point of an n-uniform affine or projective H-plane $\pi$, and let $0 \leq i \leq j \leq n$. Then $\left[{ }^{i} P: j\right]={ }^{i} P$.

Proof. Let $P \in b, P \in k$ for lines $h, k$ of $\pi$. Then Proposition 1.1 (9) implies that $b \cap{ }^{j} P=k \cap{ }^{j} P$ if and only if $|b \cap k| \geq r^{j}$. Then Proposition 1.1(5) implies that $b \cap{ }^{j} P$ is induced by $r^{n-j}$ lines of $\pi$. Then a point $Q$ of $\pi$ lies in $\left[{ }^{i} P: j\right]$ if and only if $Q$ lies in ${ }^{j} P$ and is joined to $P$ by at least $r^{j-i} \cdot r^{n-j}=$ $r^{n-i}$ lines of $\pi$. Then $Q$ is a point of $\left[{ }^{i} P: j\right]$ if and only if $Q$ is a point of ${ }^{i} P$. Since ${ }^{j} P$ and $\pi$ induce the same lines on the points of ${ }^{i} P,\left[{ }^{i} P: j\right]={ }^{i} P$.

If $P$ is a point of an affine $H$-plane $\mathcal{U}$, then $\bar{P}$ has a ready-made parallel relation induced from the parallel relation in $\mathcal{U}$. Namely, one may define $b^{P} \| g^{P}$ in $\bar{P}$ if and only if there exist lines $k, j$ in $\mathcal{Q}$ such that $b^{P}=k^{P}, g^{P}=j^{P}$, and $k \| j$ in $\mathcal{U}$. We call this the induced parallelism in $\bar{P}$. Similarly, $\mathfrak{U}$ induces an incidence structure and a parallel relation on any subset of points of $\mathfrak{U}$. In particular, if $\mathfrak{U}$ is $n$-uniform, $\mathfrak{U}$ induces a parallel relation on ${ }^{i} P$ for every point $P, 1 \leq i<n$. 
Proposition 1.3. Let $P$ be a point of $\pi$, an n-uniform affine or projective $H$ plane with invariant $r$. Then, for $1 \leq i<n,{ }^{i} P$ is an $i$-uniform affine $H$-plane with invariant $r$ (under some parallelism).

Proof. By definition of $n$-uniformity, ${ }^{n-1} P=\bar{P}$ is an $(n-1)$-uniform affine $H$ plane. Let $s, t$ denote invariants for $\pi ; s^{1}, t^{1}$, the corresponding invariants for $\bar{P}$. Then the number of points on a line of $\bar{P}$ is $s^{1}=t$ (see [7] and [9], or [3]). By Proposition 1.1(1), $t=r^{n-1}$, hence $\bar{P}$ has the invariant $r$. Now make the induction assumption that ${ }^{i+1} P$ is an $(i+1)$-uniform affine $H$-plane with the invariant $r$, some $i \leq n-2$. Then, by what we have just proved, $\left[{ }^{i} P: i+1\right]$ is an $i$-uniform affine $H$-plane with invariant $r$. The conclusion now follows by induction with the help of Lemma 1.2.

The reader is referred to [9] or [3] for the definition of translation $H$-planes as well as for most of the results on translation $H$-planes which we will use below. For the convenience of the reader we recall the following definitions. An automorphism $\tau$ of an affine $H$-plane $\mathcal{A}$ is called a translation provided: (1) $\tau$ is either the identity map or is fixed-point-free; (2) the set of fixed lines of $\tau$ is the union of complete parallel classes of 2 . A translation $H$-plane is an affine $H$-plane which possesses a point-transitive group of translations.

Proposition 1.4. Let $\mathfrak{A}$ be an n-uniform translation $H$-plane. If $P$ and $Q$ are any points of $\mathfrak{Q}$ and if $1 \leq i \leq n$, then ${ }^{i} P$ and ${ }^{i} Q$ are isomorpbic i-uniform transla. tion $H$-planes under the induced parallel relations.

Proof. Let $\bar{P}_{g}$ denote the incidence structure $\bar{P}$ together with a parallelism which makes $\bar{P}$ an $(n-1)$-uniform affine $H$-plane; let $\bar{P}_{i}$ denote $\bar{P}$ together with the induced parallelism. To prove that $\bar{P}_{i}$ is an affine $H$-plane, it suffices to show that the induced parallelism is an equivalence relation, i.e. is a parallelism, and further, that $\bar{P}_{i}$ satisfies Axiom 4 of Definition 1.1. It will follow that $\bar{P}_{i}$ is an $(n-1)$-uniform affine $H$-plane, since $\bar{P}_{g}$ is $(n-1)$-uniform and since $(n-1)$ uniformity depends only upon the incidence structure $\bar{P}$ of $\bar{P}_{g}$.

The existence of $b^{P}$ through $R$ parallel to $g^{P}$ is clear. Suppose $k^{P} \| g^{P}$ and $R \in k^{P}$. Then there exist lines $b_{1}, k_{2}, g_{1}, g_{2}$ in $\mathcal{U}$ such that $b_{1} \| g_{1}$, $k_{2} \| g_{2},\left(b_{1}\right)^{P}=b^{P},\left(k_{2}\right)^{P}=k^{P}$, and $\left(g_{1}\right)^{P}=\left(g_{2}\right)^{P}=g^{P}$. Let $\tau$ be a translation of 2 which maps some point of $g^{P}$ onto $R$. Then $\tau\left(g_{1}\right)=b_{1}$, and $\tau\left(g_{2}\right)=k_{2}$. Then it is easily seen that $b_{1} \cap k_{2}$ contains the set of all points on $b_{1}$ neighbor to $R$, i.e. the set $b_{1}^{P}=k_{2}^{P}$. Then $b^{P}=k^{P}$, and uniqueness is established.

The induced parallel relation in $\bar{P}$ is clearly reflexive and symmetric. Assume $g^{P} \| b^{P}$ and $b^{P} \| k^{P}$. Let $R \in k^{P}$. Let $g_{1}$ and $b_{1}$ be lines of $\mathscr{O}$ such that $g_{1} \| b_{1},\left(g_{1}\right)^{P}=g^{P}$, and $\left(b_{1}\right)^{P}=b^{P}$. Let $k_{1}$ be the line of $\mathscr{U}$ through $R$ which is parallel to $g_{1}$ and $b_{1}$. Then $\left(k_{1}\right)^{P}$ is the unique line parallel to $\left(g_{1}\right)^{P}$ and $\left(b_{1}\right)^{P}$ 
through $R$. Hence $\left(k_{1}\right)^{P}=k^{P}$, and $g^{P} \| k^{P}$. Then the induced parallel relation is an equivalence relation, and $\bar{P}_{i}$ is an $(n-1)$-uniform affine $H$-plane.

Since $\vec{P}_{i}$ has the parallel relation induced by $\mathscr{U}$, the neighbor translations of OU induce translations of $\bar{P}_{i}$. A neighbor translation is a translation which maps one, and hence each point, onto a point neighbor to itself [8, Satz 12]. Clearly the induced translations are transitive on the points of $\bar{P}_{i}$ and by $[8$, Satz 12], they form a group. Consequently $\vec{P}_{i}$ is a translation $H$-plane. This proves that ${ }^{n-1} P$ is an $(n-1)$-uniform translation $H$-plane under the induced parallel relation.

Assume that ${ }^{n-i} P$ is an $(n-i)$-uniform translation $H$-plane under the parallel relation induced by $\mathscr{U}$. Then ${ }^{n-i-1} P=\left[{ }^{n-i-1} P: n-i\right]$ is an $(n-i-1)$-uniform translation $H$-plane under the parallel relation induced by the parallel relation of ${ }^{n-i} P$. But if the parallel relation in ${ }^{n-i} P$ is the one induced by the parallel relation of $\mathfrak{A}$, then both ${ }^{n-i} P$ and $\mathscr{U}$ will induce the same parallel relation in ${ }^{n-i-1} P$. By induction, ${ }^{k} P$ is a $k$-uniform translation $H$-plane under the induced parallel relation for $1 \leq k \leq n$. If $\tau$ is the translation of $\mathscr{U}$ which moves $P$ to $Q$, it is easy to see that the restriction of $\tau$ to ${ }^{k} P$ is an isomorphism of ${ }^{k} P$ onto ${ }^{k} Q$.

If $\pi$ is a set of subgroups (called components) of a group $T$, we write $J(T, \pi)$ to denote the incidence structure with parallelism defined as follows: points are the elements of $T$; lines are the right cosets of the components; incidence is given by inclusion; and lines are taken to be parallel if and only if they are cosets of the same component. Lüneburg has proved the following three theorems (see [ 9 , Sätze 4.2, 4.3, 4.4, and 4.5]).

Theorem 1.5 (Lüneburg), $J(T, \pi)$ is an affine H-plane if and only if the follow. ing conditions are satisfied:

(a) The components cover $T$.

(b) If $A, B \in \pi$ and $A \cap B=1$, then $T=A B$.

(c) There exist $A, B \in \pi$ with $A \cap B=1$.

(d) The set $N=\{n \in T: n \sim 1\}$ is a normal subgroup of $T$.

(e) If $A \in \pi, A \not \subset N$.

(f) If $A \cap B=1, N=N A \cap N B$.

(g) If $A \cap B \neq 1, N A=N B$.

Theorem 1.6 (Lüneburg). If $\mathfrak{X}$ is a translation H-plane with translation group $T$, then $T$ is abelian; and there exists a collection $\pi$ of subgroups of $T$ such that $\mathfrak{Q} \simeq J(T, \pi)$. If $\mathfrak{U}=J(T, \pi)$ is an H-plane and $T$ is abelian, then $\mathfrak{X}$ is a transla. tion $H$-plane with translation group $T^{*}$ isomorpbic to $T$. The isomorphism from $T$ onto $T^{*}$ can be chosen to map $t$ to the translation $t^{*}$ defined by $t^{*}(x)=x+t$ for all $x \in T$. 
Theorem 1.7 (Lüneburg). Let $\mathfrak{X}=J(T, \pi)$ and $\mathfrak{X}^{\prime}=J\left(T^{\prime}, \pi^{\prime}\right)$ be translation $H$-planes so represented that $T$ and $T^{\prime}$ are abelian. Then $\mathfrak{A}$ and $\mathfrak{U}^{\prime}$ are isomorphic if and only if there exists a (group) isomorphism $\phi$ from $T$ onto $T^{\prime}$ such that $\phi(P) \in \pi^{\prime}$ for each $P \in \pi$.

Henceforth, we will generally require that $T$ be abelian, and we will use additive notation. If $\mathfrak{A}=J(T, \pi)$, we write ${ }^{i} T$ to denote the set of elements of $T$ which are points of ${ }^{i} 0$. The following theorem is proved in [6, Theorem 2.6(A)].

Theorem 1.8. Let $\mathfrak{U}=J(T, \pi)$ be an n-uniform translation $H$-plane with invariant $r=p^{x}, T$ abelian. Then there exist integers $l, k$ with $0 \leq l<k$ and sub. groups $C_{i}$ of $T$ which satisfy the following conditions:

(a) $T=C_{1} \oplus \cdots \oplus C_{k}$;

(b) for $i \leq l, C_{i}$ is the direct sum of $2 x$ cyclic subgroups of order $p^{j+1}$, $j>0$

(c) for $i>l, C_{i}$ is the direct sum of $2 x$ cyclic subgroups of order $p^{j}$;

(d) for $i \leq n=k j+l$,

$$
{ }^{i} T=p^{q+1} \cdot\left(C_{1} \oplus \cdots \oplus C_{e}\right) \oplus p^{q} \cdot\left(C_{e+1} \oplus \cdots \oplus C_{k}\right)
$$

where $q$, $e$ are given by $n-i=k q+e, 0 \leq e<k$.

Definition 1.5. Let $\mathfrak{U}=J(T, \pi)$ be an $n$-uniform translation $H$-plane with $T$ as in Theorem 1.8. Then we call $k$ the width of $\mathfrak{A}$. If $k=1$, we say that $\mathfrak{A}$ is a vertical $n$-uniform translation $H$-plane. If $k=n$ or equivalently, if $T$ is elementary abelian, we say that $\mathcal{O}$ is borizontal.

Remark 1.9. For an $n$-uniform translation $H$-plane $\mathfrak{U}=J(T, \pi)$ with translation group $T^{*}$, the following are equivalent:

(a) 2 is vertical;

(b) $T^{*}$ is the direct sum of cyclic subgroups of common order $p^{n}, p$ prime;

(c) $p T^{*}$ is the group of neighbor translations of 2 .

Proof. A neighbor translation is a translation which maps some point and hence each point (see [8, Satz 12]) of an affine $H$-plane onto a point neighbor to itse lf. Let $t^{*}: T \rightarrow T, x \rightarrow x+t$. Let $\left({ }^{n-1} T\right)^{*}=\left\{t^{*}: t \in \epsilon^{n-1} T\right\}$. Then $\left({ }^{n-1} T\right)^{*}$ is clearly the group of neighbor translations of $\mathcal{U}$. The equivalence of (a) and (c) follows from Theorem 1.8. It is well known that the number of points in an affine $H$-plane is $s^{2}$ which by Proposition 1.1(1) is $r^{2 n}$. Now $\mathscr{O}$ is vertical if and only if $o(T)=o\left(C_{1}\right)$, i.e. if and only if $r^{2 n}=\left(p^{j}\right)^{2 x}$, i.e. if and only if $n=j$ which is equivalent to condition (b).

In light of Proposition 1.4, one can investigate all neighborhoods ${ }^{i} X$ for all 
points $X$ of an $n$-uniform translation $H$-plane by investigating all neighborhoods ${ }^{i} P$ of a single point $P$. Therefore, we introduce the following notation. If $\mathfrak{U}=$ $J(T, \pi), T$ abelian, we write ${ }^{i} \mathscr{X}$ to denote the incidence structure ${ }^{i} 0$ together with the parallel relation induced therein by $\mathscr{X}$. Theorem 1.8 implies that ${ }^{i} T$ is a subgroup of $T$ for $0 \leq i \leq n$. It is easy to see that ${ }^{i \mathscr{Q}}=J\left({ }^{i} T,{ }^{i} \pi\right)$ where ${ }^{i} \pi=$ $\left\{P \cap{ }^{i} T: P \in \pi\right\}$. We will also write $T_{j}$ to denote $T /{ }^{j} T, \pi_{j}$ to denote $\left\{\left(P+{ }^{j} T\right) /{ }^{j} T: P \in \pi\right\}$, and $\mathscr{U}_{j}$ to denote $J\left(T_{j}, \pi_{j}\right)$.

As in the preceding paragraph, the letters $P, Q$ and $R$ will later be used to denote both points of an H-plane and components of a "generalized congruence" $\pi$. We hope to avoid confusion by indicating the use of the letter at the time of introduction. In addition, on those occasions when we are looking at an $H$-plane represented in the form $J(T, \pi)$, we use lower case Roman letters, a, b, c, . . to denote points (group elements).

Proposition 1.10. Let $\mathfrak{U}=J(T, \pi)$ be an n-uniform translation $H$-plane represented so that $T$ is abelian. Then

(a) îf is an i-uniform translation $H$-plane for $1 \leq i \leq n$.

(b) The canonical (group) bomomorphism from ${ }^{i} T$ onto ${ }^{i} T /{ }^{j} T$ induces an epimorpbism from in to (iथ) ${ }_{j}$ for $0 \leq j<i \leq n$.

(c) If $\mathfrak{Q}$ has width $k$, then $(\mathrm{iQU})_{j}$ and $\left({ }^{i-k \mathfrak{U})_{j-k}}\right.$ are isomorphic when $k \leq$ $j<i \leq n$.

Proof. Conclusion (a) is just a special case of Proposition 1.4, so there is nothing new to prove here. By Lemma $1.2,{ }^{j}\left({ }^{i} T\right)={ }^{j} T$. To prove (b), let $\phi$ be the natural (group) homomorphism from ${ }^{i} T$ onto ${ }^{i} T /{ }^{i} T$. Observe that $\phi$ maps each component in ${ }^{i} \pi$ onto a component in $\left({ }^{i} \pi\right)_{j}$ and that each component in $\left({ }^{i} \pi\right)_{j}$ is the image under $\phi$ of some component in ${ }^{i} \pi$. It is clear then that $\phi$ induces an epimorphism from iथ to $\left({ }^{i} \mathscr{U}\right)_{j}$.

We prove (c) first in the special case $i=n$. Let $\phi, \phi^{*}$ be the respective natural homomorphisms from $T$ onto $T /{ }^{j} T$ and from ${ }^{n-k} T$ onto ${ }^{n-k} T /{ }^{j-k} T$. By Theorem 1.8, we know that $T$ is a $p$-group for some prime $p$ and that the map $\psi$ defined on $T$ by $\psi(t)=p t$ is a group homomorphism onto ${ }^{n-k} T$. We define $\mu$ from $T /{ }^{j} T$ to ${ }^{n-k} T /{ }^{j-k} T$ by the rule $\mu\left(t+{ }^{j} T\right)=\phi^{*} \psi(t)$. By Theorem 1,8 , we know that $\mu$ is a well-defined (group) isomorphism of $T /{ }^{j} T$ onto ${ }^{n-k} T /{ }^{j-k} T$. We summarize the maps defined above in the following commutative diagram.

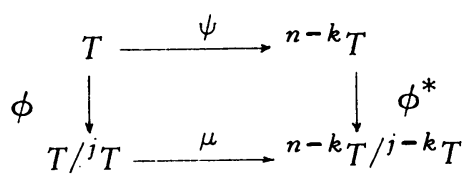


Let $R \in \pi$. Then $\psi(R)=p R \subset R \cap{ }^{n-k} T$. We give a counting argument to prove that $\psi(R)=R \cap{ }^{n-k} T$. There is a component $S$ in $\pi$ such that $R \cap S=0$. By Theorem 1.5(b), $R+S=T$. By Lüneburg [9, Korollar 4.1], $R \cong S$. Then $R$ has half as many summands as $T$. Since $o(\operatorname{Ker} \psi)=r^{2 k}, o(\operatorname{Ker} \psi \cap R)=r^{k}$. Then

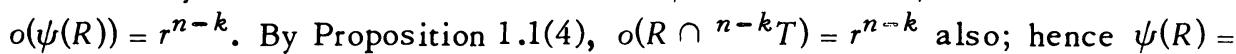
$R \cap{ }^{n-k} T$. Then $\mu\left[\left(R+{ }^{j} T\right) /{ }^{j} T\right]=\phi^{*} \psi(R)=\phi^{*}\left(R \cap{ }^{n-k} T\right)$. Since $R \cap{ }^{n-k} T$ is a component in ${ }^{n-k} \pi, \mu$ maps each component in $\pi_{j}$ onto a component of $\left({ }^{n-k} \pi\right)_{j-k}$. Since every component in ${ }^{n-k} \pi$ is of the form $R \cap{ }^{n-k} T$ for some $R$ in $\pi, \mu$ maps the set of components of $\pi_{j}$ onto the set of all components of $\left({ }^{n-k} \pi\right)_{j-k}$. Then it is clear that $\mu$ induces an isomorphism from $\mathfrak{A}_{j}$ onto $\left({ }^{n-k \mathscr{U}}\right)_{j-k}$. Now let $k \leq j<i \leq n$. By (a), iथ is an $i$-uniform translation $H$-plane. From Theorem 1.8, we see that iी has width $k$. Then the full result (c) follows from the case $i=n$ which we have just proved.

For $n \geq i \geq 1$, we write $\mathscr{U}(i)$ to denote $\left.{ }^{(i \mathfrak{Q}}\right)_{i-1}$. Clearly, $\mathscr{U}(i)$ is the ordinary affine plane which is canonically associated with ${ }^{i \mathscr{U}}$. Since ${ }^{i \mathfrak{U}}$ is a translation $H$ plane, $\mathscr{U}(i)$ is a customary translation plane. Thus we have associated with each $n$-uniform translation $H$-plane $\mathfrak{U}$ a sequence $\mathfrak{U}(1), \ldots, \mathfrak{U}(n)$ of $n$ ordinary affine translation planes. As a special case of Proposition $1.10(\mathrm{c})$, we have

Remark 1.11. Let $\mathfrak{A}$ be an $n$-uniform translation $H$-plane of width $k$. Then the sequence of associated translation planes, $\mathscr{U}(1), \ldots, \mathfrak{U}(n)$, is periodic with a period length dividing $k$. In particular, if $\mathfrak{X}$ is vertical, $\mathfrak{X}(1) \cong \mathfrak{X}(2) \cong \ldots \cong$ $\mathfrak{U}(n)$.

We shall see in $\$ 6$ that $\mathfrak{U}(1), \ldots, \mathfrak{U}(k)$ may be arbitrary translation planes of order $r, r$ being the invariant of $\mathfrak{U}$.

2. Strongly $n$-uniform translation $H$-planes. We begin with some notation. If $P$ is a point and $b$ is a line of an $n$-uniform $H$-plane, we write $P(\sim i) b$ (and say $P$ is at least $i$-related to $b)$ to mean that $P(\sim i) Q$ for some point $Q$ on $b$; otherwise we write $P(\mathcal{L} i) b$. We write $P\left(\simeq_{i}\right) b$ (and say $P$ is $i$-related to $b$ ) if $P(\sim i) b$ and $P(\not i+1) b$.

Definition 2.1. Let $\pi$ be an $n$-uniform $H$-plane. We say $\pi$ has Property $S$ (called Property $A$ in [4]) provided that $Q(\simeq i+j) k$ whenever $P, Q, b, k$ are points and lines of $\pi$ satisfying the following requirements: $|b \cap k|=r^{i}, P \in$ $b \cap k, Q \in b-k, Q(\simeq j) P$, and $i+j<n$.

In [4], strongly $n$-uniform projective $H$-planes are defined and then characterized in four additional ways. One characterization (see [4, Theorem 4.2]) is as the class of all $n$-uniform projective $H$-planes with Property S. We now define strongly n-uniform affine $H$-planes to be the $n$-uniform affine $H$-planes which satisfy Property S. Some justification of this definition is in order. For if an affine $H$-plane $\mathscr{U}$ is obtained from a projective $H$-plane $\pi$ by removing a neighbor 
class of lines (see Klingenberg [8, p. 101] or Dembowski [3] for particulars), many pairs of lines $\{b, k\}$ will fail to intersect in $\mathcal{U}$. Suppose that $|b \cap k \cap \mathfrak{U}|=0$, that $b \cap k$ contains a point $P$ in $\pi$, and that $\pi$ is strongly $n$-uniform. Since every affine point $Q$ on $b$ satisfies $Q(\simeq 0) P$, every such $Q$ must satisfy $Q(\simeq i) k$ where $r^{i}=|b \cap k|$ in $\pi$. Thus it seems natural to require strongly $n$-uniform affine $H$ planes to satisfy both Property $S$ and the Property $S^{\prime}$ stated below.

Definition 2.2. Let $\mathfrak{U}$ be an $n$-uniform affine $H$-plane. We say $\mathfrak{U}$ has Property $\mathrm{S}^{\prime}$ if $|b \cap k|=0$ implies the existence of an integer $i$ such that $Q(\simeq i) k$ for all points $Q$ on $b$.

The following result justifies the definition given above for strongly $n$-uniform affine $H$-planes.

Remark 2.1. Every strongly $n$-uniform affine $H$-plane has Property $\mathrm{S}^{\prime}$.

Proof. Let $\mathfrak{A}$ be a strongly $n$-uniform affine $H$-plane. Assume $\mathfrak{A}$ does not have Property $\mathrm{S}^{\prime}$; then there exist lines $b, k$ and points $P, Q \in b$ such that $P(\simeq i) k, Q(\simeq j) k,|b \cap k|=0$, and $n>i>j \geq 0$. Since $b$ contains a pair of nonneighbor points, we may assume without loss of generality that $P \not Q$. Let $\phi$ be the canonical epimorphism from $\mathfrak{U}$ onto its associated ordinary affine plane. Since $|b \cap k|=0, b^{\phi} \| k^{\phi}$ by Axiom 3c of Definition 1.1. Since $P^{\phi}$ is on both $b^{\phi}$ and $k^{\phi}, b^{\phi}=k^{\phi}$, hence $b \sim k$. Then $Q(\sim 1) k$, so $j>0$.

We set

$$
x^{*}=\left\{x: P \in x,|x \cap k|=r^{j}\right\}, \quad X^{*}=\{X \in k: X(\simeq i-j) P\} .
$$

Let $X \in x \cap k$ for some $x \in x^{*}$. Then Property $S$ implies $X(\simeq i-j)$, i.e. $X \in$ $X^{*}$. Conversely, if $X \in X^{*}$ and if $x$ is a line containing $X$ and $P$, Property $\mathrm{S}$ implies $x \in x^{*}$. Let $P^{\prime}$ be a point of $k$ such that $P^{\prime}(\simeq i) P$. Since $j>0$, Proposition 1.1(6) implies $X(\simeq i-j) P$ if and only if $X(\simeq i-j) P^{\prime}$. Then Proposition 1.1(4) implies $\left|X^{*}\right|=r^{n-i+j}-r^{n-i+j-1}$. We see that each $X \in X^{*}$ is joined to $P$ by $r^{i-j}$ lines of $x^{*}$ and that each $x \in x^{*}$ joins $r^{j}$ points of $X^{*}$ to $P$. Then

$$
\left|x^{*}\right|=\left(r^{n-i+j}-r^{n-i+j-1}\right) r^{i-j} / r^{j}=r^{n-j}-r^{n-j-1} \text {. }
$$

By [9, Satz 2.3], we know that there exists a line $b$ through $Q$ such that $b \nsim b, b \nsim k$. If $|b \cap k|=0, b^{\phi} \| k^{\phi}$; and since $Q^{\phi}$ is on both $b^{\phi}$ and $k^{\phi}, b^{\phi}=$ $k^{\phi}$. We obtain the contradiction $b \sim k$, hence conclude that $b \cap k$ must contain a point $R$. Suppose $R(\simeq l) Q$. If $l<j$, either Property S or Proposition 1.1(7) would imply $b \sim k_{\text {. Then }} R(\simeq j) Q$. Now let $x \in x^{*}$. If $|b \cap x|=0, b^{\phi} \| x^{\phi}=k^{\phi}$; hence $b^{\phi}=k^{\phi}$; hence $b \sim k$, a contradiction. Then $|b \cap x| \geq 1$. Since $x \sim k, x \not b$; hence $|b \cap x|=1$. There exists $X \in x \cap k$ such that $X \sim P \not Q \sim R$. Since $R \nsim X$, Property S implies $R(\simeq j) x$. Then $R(\simeq l)(b \cap x), l \leq j$. If $l<j$, Property $S$ would imply $b \sim x$, hence $b \sim k$. By the contradiction, $l=j$ and $R(\simeq j)(b \cap x)$. 
Since $(b \cap x) \sim Q,(b \cap x) \not P$. Then if $b \cap x=b \cap x^{\prime}$ for $x^{\prime} \in x^{*}, x=x^{\prime}$. Then $\left\{b \cap x: x \in x^{*}\right\}$ contains all of the $r^{n-j}-r^{n-j-1}$ points $S$ of $b$ satisfying $S(\simeq j) R$. In particular, $Q=b \cap x$ for some $x \in x^{*}$. Then $b$ and $x$ both contain $Q$ and $P$; hence $b=x$ and $|b \cap k|>0$. This contradiction completes the proof that Of has Property $S^{\prime}$.

Proposition 2.2. Let $\pi$ be a strongly $n$-uniform affine or projective $H$-plane. If $P$ is a point of $\pi$ and if $1 \leq i<n$, then ${ }^{i} P$ is a strongly $i$-uniform affine $H$ plane for every parallel relation which makes ${ }^{i} P$ an affine $H$-plane.

Proof. Since $\pi$ is $n$-uniform, ${ }^{n-1} P$ is an $(n-1)$-uniform affine $H$-plane for some parallel relation. Then ${ }^{n-1} P$ is $(n-1)$-uniform under every parallel relation which makes ${ }^{n-1} P$ an affine $H$-plane. Henceforth, let $\bar{P}$ denote the incidence structure ${ }^{n-1} P$ together with a fixed parallel relation which makes ${ }^{n-1} P$ an affine $H$-plane. Let $Q, R, b^{P}, k^{P}$ be points and lines of $\bar{P}$ such that $\left|b^{P} \cap k^{P}\right|=$ $r^{i}, Q \in b^{P} \cap k^{P}, R \in b^{P}-k^{P}, R(\simeq j) Q$ in $\bar{P}$, and $0 \leq j<n-1-i$. Then $|b \cap k|=r^{i}, Q \in b \cap k, R \in b-k, R(\simeq j+1) Q$ in $\pi$, and $0<j+1<n-i$. Then Property $S$ implies $R(\simeq i+j+1) k$ in $\pi$. Clearly, any point $T$ on $k$ such that $R(\simeq i+j+1)) T$ lies in $k^{P}$. Then $R(\simeq i+j) k^{P}$ in $\bar{P}$. Thus $\bar{P}$ satisfies Property $S$, hence is strongly $(n-1)$-uniform. The full result now follows by induction and the use of Lemma 1.2 with $j=i+1$.

Proposition 2.3. Let $\mathfrak{Q}$ be an affine $H$-plane belonging to a strongly $n$-uniform projective H-plane $\pi$. If $P$ is a point of $\mathscr{U}$ and if $1 \leq i<n$, then ${ }^{i} P$ is a strongly $i$-uniform affine $H$-plane under the induced parallelism.

Proof. By a theorem of Artmann (see [2, Satz 4] and Bacon [10]), all strongly $n$-uniform projective $H$-planes are of height $n$ and satisfy "Axiom N". The author has proved [5, Theorem 8.1] that every point $P$ of every affine $H$-plane $\mathcal{A}$ belonging to such a projective $H$-plane is "nilpotent of degree $n$ ". By definition [5, Definition 7.3], this implies that $P_{n-i}=\left[{ }^{i} P: i+1\right]$ is an affine $H$-plane (see [5, Definition 3.4]) under the parallelism induced by $\mathfrak{U}$. By Lemma $1.2,{ }^{i} P=$ $P_{n-i}$, and the full conclusion now follows from Proposition 2.2.

Combining Propositions 1.4 and 2.2, we obtain

Proposition 2.4. Let $\mathfrak{A}$ be a strongly $n$-uniform translation $H$-plane. If $P$ and $Q$ are points of $\mathscr{U}$ and if $1 \leq i \leq n$, then ${ }^{i} P$ and ${ }^{i} Q$ are isomorpbic strongly $i$ uniform translation $H$-planes under the parallel relations induced by $\mathfrak{A}$.

Lemma 2.5. Let $J(T, \pi)$ be a strongly $n$-uniform affine $H$-plane with invariant r. Let $P, Q \in \pi, o(P \cap Q)=r^{i}, b \in P, b(\simeq j) 0$ where $i$ and $j$ are nonnegative integers satisfying $i+j<n$. Then $b \in^{n-i-j} T+Q, b \notin^{n-i-j-1} T+Q$. 
Proof. Property $\mathrm{S}$ implies the existence of $d \in Q$ such that $b(\simeq i+j) d$. Then there are exactly $r^{i+j}$ cosets $R+d$ with $R \in \pi$ which contain both $b$ and $d$. Then $b-d$ lies in exactly $r^{i+j}$ components $R$, i.e. $(b-d)(\simeq i+j) 0$, i.e. $(b-d) \epsilon^{n-i-j} T$. Then $b \epsilon^{n-i-j} T+Q$. The second conclusion is also easy to see.

As an immediate consequence of Lemma 2.5, we obtain

Corollary 2.6. Let $J(T, \pi)$ be a strongly $n$-uniform affine $H$-plane with invariant r. Let $P, Q \in \pi, o(P \cap Q)=r^{i}$ for some nonnegative $i<n$. Then $P \subset{ }^{n-i} T+$ $Q, P \not \subset^{n-i-1} T+Q$.

In Proposition 1.10, we examined some incidence structures with parallelisms-the $\left({ }^{(\mathfrak{U}}\right)_{j}$ associated with an $n$-uniform translation $H$-plane $\mathfrak{A}$. We will prove in Proposition 2.9 that if $\mathscr{U}$ is strongly $n$-uniform and if $0 \leq j<i \leq n$, then $(i \mathfrak{U})_{j}$ is an affine $H$-plane. To aid in the proof of this result and also to facilitate the further study of the $\left({ }^{i}()_{j}\right)$ in $\$ 6$, we prove two additional corollaries to Lemma 2.5. To state these corollaries, we introduce the following notation. Let $\mathscr{U}=$ $J(T, \pi)$ be an $n$-uniform translation $H$-plane with invariant $r, T$ abelian. Then we write $\left(d+{ }^{j} T\right)(\sim i)\left(b+{ }^{j} T\right)$ to mean that $d+{ }^{j} T$ and $b+{ }^{j} T$ are joined by at least $r^{i}$ lines of $\mathfrak{U}_{j}$. We write $(\simeq i)$ to indicate that two points are joined by exactly $r^{i}$ lines of $\mathfrak{U}_{j}^{j}$.

Corollary 2.7. Let $J(T, \pi)$ be a strongly $n$-uniform translation $H$-plane with invariant $r, T$ abelian. Then for $i<n-j,\left(d+{ }^{j} T\right)(\simeq i)\left(b+{ }^{j} T\right)$ if and only if $d(\simeq i) b$.

Proof. Let $a \in Q \in \pi, a(\simeq i) 0$ for some $i<n-j$. Then $\left(a+{ }^{j} T\right) \epsilon$ $\left(P+{ }^{j} T\right) /{ }^{j} T$ for $P \in \pi$ if and only if $a \in P+{ }^{j} T$, i.e. (by Lemma 2.5) if and only if $o(P \cap Q) \geq r^{n-j-i}$. By Proposition 1.1(5), the number of such $P \in \pi$ is $r^{i+j}$. Next, we compute the number $m$ of $R \in \pi$ satisfying $\left(R+{ }^{j} T\right) /{ }^{j} T=\left(P+{ }^{j} T\right) /{ }^{j} T$ for a fixed $P \in \pi$. Clearly, $m$ is the number of $R \subset P+{ }^{j} T$. By Corollary $2.6, m$ is the number of $R$ satisfying $o(R \cap P) \geq r^{n-j}$; hence $m=r^{j}$. Then $a+{ }^{j} T$ and ${ }^{j} T$ are joined by $r^{i+j} / m=r^{i}$ lines of $A_{j}$, i.e. $\left(a+{ }^{j} T\right)(\simeq i){ }^{j} T$. We have $\left(d+{ }^{j} T\right)$ $(\simeq i)\left(b+{ }^{i} T\right)$ if and only if $\left(d-b+{ }^{j} T\right)(\simeq i){ }^{j} T$, i.e. if and only if $(d-b)$ $(\simeq i) 0$ which is true if and only if $d(\simeq i) b$.

Corollary 2.8. Let $J(T, \pi)$ be a strongly $n$-uniform translation $H$-plane with invariant $r, T$ abelian. Let $P, Q \in \pi$; let $0 \leq j<n$. Then

(a) $P+{ }^{j} T=Q+{ }^{j} T \Leftrightarrow o(P \cap Q) \geq r^{n-j}$;

(b) $o\left[\left(\left(P+{ }^{j} T\right) /{ }^{j} T\right) \cap\left(\left(Q+{ }^{j} T\right) /{ }^{j} T\right)\right]=o(P \cap Q)$ if $o(P \cap Q) \leq r^{n-j}$.

Proof. The truth of (a) follows immediately from Corollary 2.6. Now assume 
$o(P \cap Q)=r^{i}, \quad i \leq n-j$. The points common to $\left(P+{ }^{j} T\right) /{ }^{j} T$ and $\left(Q+{ }^{j} T\right) /{ }^{j} T$ are the cosets $b+{ }^{j} T$ with $b \in P \cap\left(Q+{ }^{j} T\right)$. By Lemma 2.5, these are the points $b+{ }^{j} T$ with $b \in P$ such that $b(\sim n-j-i) 0$. By Proposition 1.1(4), the number of such $b$ is $r^{j+i}$; hence the number of such $b+{ }^{j} T$ is $r^{i}$.

Proposition 2.9. If $2 \mathbb{C}$ is a strongly n-uniform translation $H$-plane, then $\left.{ }^{(i 2}\right)_{j}$ is a strongly $(i-j)$-uniform translation $H$-plane for $0 \leq j<i \leq n$.

Proof. In view of Proposition 2.4, it suffices to prove the result for $\left(n_{\mathfrak{U}}\right)_{j}=$ $\mathfrak{U}_{j}, 0 \leq j<n$. We represent $\mathfrak{U}$ by $J(T, \pi)$ with $T$ abelian. Writing $\left(d+{ }^{j} T\right) \sim$ $\left(b+{ }^{j} T\right)$ to mean that $d+{ }^{j} T$ and $b+{ }^{j} T$ are joined by at least two lines of $\mathfrak{Q}_{j}$ and setting $i=0$ in Corollary 2.7 , we obtain

$$
\left(d+{ }^{j} T\right) \sim\left(b+{ }^{j} T\right) \Leftrightarrow d \sim b .
$$

It follows easily from (2.1), Corollary $2.8(\mathrm{~b})$, and Theorem 1.5 that $\mathfrak{A}_{j}$ is an affine $H$-plane. Since $T$ is abelian, so is $T_{j}$, and thus Theorem 1.6 implies that $\mathscr{N}_{j}$ is even a translation $H$-plane.

Next, we give a proof by induction on $n$ to show that $\mathfrak{U}_{j}$ is $(n-j)$-uniform. If $j<n-1$, the induction assumption and Proposition 2.4 imply that $(n-1 \mathscr{Q})_{j}$ is an $(n-1-j)$-uniform translation $H$-plane. We let $\$$ denote the set of points $b+{ }^{j} T$ of $\mathfrak{U}_{j}$ which are neighbor in $\mathscr{U}_{j}$ to ${ }^{j} T$. We write $\left(\mathfrak{U}_{j}\right)^{*}$ to denote the incidence structure induced in $\Re$ by $\mathscr{U}_{j}$. We wish to prove that $\left(\mathscr{U}_{j}\right)^{*}$ is the incidence structure of $\left({ }^{n-1} \mathfrak{U}\right)_{j}$. By $(2.1)$, the points of $\left(\mathscr{R}_{j}\right)^{*}$ are the $b+{ }^{j} T$ with $b \sim 0$, i.e. the $b+{ }^{j} T$ with $b \in e^{n-1} T$. The points of $\left({ }^{n-1} \mathfrak{U}\right)_{j}$ are the elements of ${ }^{n-1} T /^{j(n-1)} T$. By Lemma 1.2, ${ }^{j(n-1)} T={ }^{j} T$. Thus $\left({ }^{n-1} \mathfrak{U}\right)_{j}$ and $\left(\mathscr{U}_{j}\right)^{*}$ have the same point set. Using $(2.1)$, it is easy to see that $\left(\mathscr{U}_{j}\right)^{*}$ and $\left({ }^{n-1} \mathfrak{U}_{j}\right)_{j}$ also have the same lines, hence are isomorphic qua incidence structures. Since $\mathfrak{A}_{j}$ is a translation $H$-plane, the neighborhood of every point of $\mathscr{U}_{j}$ is isomorphic to $\left(\mathscr{U}_{j}\right)^{*}$, hence is isomorphic qua incidence structure to $\left({ }^{n-1} \mathfrak{U}\right)_{j}^{j}$, hence is $(n-1-j)$-uniform.

Corollary 2.8(a) and Proposition 1.1(5) imply

$$
\left|\left\{X \in \pi: X+{ }^{j} T=P+{ }^{j} T\right\}\right|=r^{j} .
$$

For $P \in \pi$, let $P^{*}$ denote $\left(P+{ }^{j} T\right) \cap{ }^{n-1} T$. Then $P^{*} \subset Q^{*}$ if and only if $\left(P \cap{ }^{n-1} T\right) \subset\left(Q+{ }^{j} T\right)$. Suppose $o(P \cap Q)=r^{l}$. Since

$$
\left(P \cap{ }^{n-1} T\right)=\{b \in P: b(\simeq i) 0, i \geq 1\}
$$

Lemma 2.5 implies that $\left(P \cap{ }^{n-1} T\right) \subset Q+{ }^{j} T$ if and only if $j \geq n-l-1$, i.e. if and only if $l \geq n-j-1$. By symmetry, $P^{*}=Q^{*}$ if and only if $P^{*} \subset Q^{*}$. From Proposition 1.1(5), we obta in 


$$
\left|\left\{P \in \pi: P^{*}=Q^{*}\right\}\right|=r^{j+1} \text { for } Q \in \pi .
$$

Each line of $\left(\mathscr{R}_{j}\right)^{*}$ which contains the point ${ }^{j} T$ is of the form

$$
\left(\left(Q+{ }^{j} T\right) /{ }^{i} T\right) \cap\left({ }^{n-1} T /{ }^{j} T\right)=Q^{*} /{ }^{j} T
$$

with $Q \in \pi$. From (2.2) and (2.3), we conclude that each such line is the restriction of $r$ distinct lines of $\mathfrak{Q}_{j}$. Since $\mathfrak{Q}_{j}$ is a translation $H$-plane, every line of every point neighborhood in $\mathfrak{U}_{j}$ is the restriction of $r$ lines of $\mathscr{U}_{j}$. Then $\mathfrak{U}_{j}$ is $(n-j)$-uniform if $j<n-1$.

By Theorem $1.5(\mathrm{f}),(\mathrm{g})$, we see that distinct lines of $\mathfrak{O H}_{n-1}$ which contain the point ${ }^{n-1} T$ have trivial intersections. Then $\mathscr{U}_{n-1}$ is an affine $H$-plane one of whose points has no neighbors distinct from itself. Thus $\mathfrak{A}_{n-1}$ is an ordinary affine plane, i.e. is a 1 -uniform affine $H$-plane. This completes the induction proof that $\mathfrak{U}_{j}$ is $(n-j)$-uniform.

All that remains is to verify that the $\mathfrak{U}_{j}$ possess Property S. Assume $\left(P+{ }^{j} T\right) /{ }^{i} T$ and $\left(Q+{ }^{i} T\right) /{ }^{j} T$ meet in $r^{i}$ points for some $i<n-j$ and that $b+{ }^{j} T$ is a point of $\left(P+{ }^{j} T\right) /{ }^{j} T$ such that $\left(b+{ }^{j} T\right)(\simeq k){ }^{j} T$ for some $k<n-j-i$. Then there exists $t \in{ }^{j} T$ such that $b^{\prime}=b+t \in P$. Then $b^{\prime}(\sim n-j) b$, and by Corollary 2.7, $b(\simeq k)$ 0. By Proposition 1.1(6), $b^{\prime}(\simeq k) 0$. By Corollary 2.8(b), $o(P \cap Q)=r^{i}$. Since $\mathcal{A}$ possesses Property $S, b^{\prime}(\simeq i+k) Q$. Since $b^{\prime}+{ }^{j} T=$ $b+{ }^{j} T$, Corollary 2.7 implies

$$
\left(b+{ }^{j} T\right)(\simeq i+k)\left(Q+{ }^{j} T\right) /{ }^{j} T .
$$

This is the desired result. That the $\mathscr{U}_{j}$ satisfy Property $S$ for points and lines in general position follows from the fact that the $\mathscr{U}_{j}$ are translation $H$-planes.

3. The constructions. The constructions of $n$-uniform translation $H$-planes given in this section make extensive use of group "congruences". A congruence of a group $T$ is a partition $\pi$ of $T$ into proper subgroups such that $T=B C$ whenever $B \neq C$ and $B, C \in \pi$. André has proved [1, pp. 164-167] that if $\pi$ is a congruence of $T$, then $J(T, \pi)$ is an ordinary translation plane with translation group isomorphic to $T$; and conversely, that every translation plane $A$ is isomorphic to $J(T, \pi)$ where $\pi$ is a congruence of $T$ and $T$ is the translation group of 2 . It follows that a finite group $T$ has a congruence if and only if $T$ is an elementary abelian p-group for some prime $p$ and the order of $T$ is a square. If a group $T$ of order $r^{2}$ has a congruence, one may obtain a congruence for $T$ containing any specified subgroup of order $r=p^{x}$ as one of its components. To see this, regard $T$ as a vector space over $G F(p)$, and let $\phi$ be a linear automorphism of $T$ mapping a component of the first congruence onto the desired subgroup. Lastly, we observe that the following conditions imply that $\pi$ is a congruence of $T: T$ is a group of order $r^{2} ; \pi$ is a collection of $r+1$ subgroups of $T$, each of order $r$; and $A \cap B=1$ when $A, B \in \pi, A \neq B$. 
Before proceeding to a description of the constructions, we prove the following

Lemma 3.1. Let $\mathfrak{A}=J(T, \pi)$ be an n-uniform translation $H$-plane with invariant $r, T$ abelian. Then if $P \in \pi$ and if $0 \leq i \leq n$,

(a) $o\left(P \cap{ }^{i} T\right)=r^{i}$

(b) $o\left(P+{ }^{i} T\right)=r^{n+i}$

Proof. The truth of (a) is a consequence of Proposition 1.1(4). By Theorem $1.8,{ }^{i} T$ is a subgroup of $T$. By Proposition 1.1(8), o $\left({ }^{i} T\right)=r^{2 i}$ (unless $i=n$, but it is well known that the number of points in a finite affine $H$-plane is $s^{2}=r^{2 n}$ ). The truth of (b) is now clear.

We are now ready to describe the constructions. Let $\mathfrak{U}=J(T, \pi)$ be an $n$ uniform translation $H$-plane with invariant $r=p^{x}$ and translation group isomorphic to $T$. We write

$$
T=C_{1} \oplus \cdots \oplus C_{k},
$$

where $C_{i}, j, k, l$ have the same meaning as in Theorem 1.8. We set

$$
T^{\#}=D_{1} \oplus \cdots \oplus D_{k},
$$

where $D_{i}=C_{i}$ for $i<k, D_{k}$ is a direct sum of $2 x$ cyclic groups of order $p^{j+1}$, and $p D_{k}=C_{k}$. If $T$ is elementary abelian, then $l=0, j=1, k=n$, and each $C_{i}$ is a direct sum of $2 x$ cyclic groups of order $p$. In this case, we set

$$
T^{e}=C_{0} \oplus \cdots \oplus C_{k}
$$

where $C_{0} \cong C_{1}$.

Our goal is to define collections $\pi^{\#}, \pi^{e}$ of subgroups so that $J\left(T^{\#}, \pi^{\sharp}\right)$ and $J\left(T^{e}, \pi^{e}\right)$ will be $(n+1)$-uniform translation $H$-planes. Let $m$ be a positive integer $<n$. Let $Q, R, S \in \pi$ be such that $o(Q \cap R) \geq r^{m}$ and $o(R \cap S) \geq r^{m}$. By Proposition 1.1(9), $Q$ and $S$ both contain all points $x \in R$ such that $x(\sim n-m)$

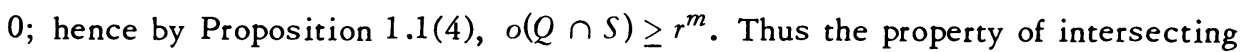
in $r^{m}$ or more points induces an equivalence relation on $\pi$. (If $m=n$, the trivial equivalence relation occurs.) We now take $\rho$ to be a subset of $\pi$ such that, for each $S \in \pi$, there is a unique $R \in \rho$ which satisfies $o(S \cap R) \geq r^{n+1-k}$. Proposition 1.1(5) tells us that each $R \in \rho$ intersects exactly $r^{k-1}$ components of $\pi$ in $r^{n+1-k}$ or more points. These components, we denote by the labels

$$
R\left(\alpha_{1}, \cdots, \alpha_{k-1}\right), \quad 0 \leq \alpha_{i}<r \text { for all } i
$$

For convenience, we always set $R(0, \ldots, 0)=R$. By Proposition 1.1(5), (9), we may arrange the labels $R\left(\alpha_{1}, \ldots, \alpha_{k-1}\right)$ so that 


$$
R\left(\alpha_{1}, \ldots, \alpha_{k-1}\right) \cap R\left(\beta_{1}, \cdots, \beta_{k-1}\right)={ }^{n-k+i} T \cap R\left(\alpha_{1}, \ldots, \alpha_{k-1}\right),
$$

where $i$ is the largest integer $\leq k$ such that $\alpha_{l}=\beta_{l}$ for all $l<i$. Note that if $k=1$, then $\rho=\pi$, and $R()=R$ for all $R \in \rho$.

Let $R^{\#}=\left\{t \in T^{\#}: p t \in R\right\}$. By Theorem 1.8(d), $p R^{\#}={ }^{n+1-k} T \cap R$. Then using Lemma 3.1, one obtains $o\left(R^{\#}\right)=p^{2 x k} \cdot o\left(p R^{\#}\right)=r^{2 k} \cdot r^{n+1-k}=r^{n+1+k}$. Clearly, ${ }^{k-1} T+R \subset{ }^{k} T+R \subset R^{\#}$. By Lemma 3.1, o $\left({ }^{k} T+R\right)=r^{n+k}$, and $o\left({ }^{k-1} T+R\right)=$ $r^{n+k-1}$. Since $R^{\#} /\left({ }^{k-1} T+R\right)$ is elementary abelian of order $r^{2}$, it has a congruence, one of whose components is

$$
\left({ }^{k} T+R\right) /\left({ }^{k-1} T+R\right) .
$$

The remaining components, we denote by

$$
R[\alpha] /\left({ }^{k-1} T+R\right), \quad 0 \leq \alpha<r .
$$

If $k=1$, we set $\pi^{\#}=\{R[\alpha]\}$.

To construct an $H$-plane in the elementary abelian group $T^{e}$, we must begin somewhat differently. We first observe that $C_{0}$ has a congruence $\pi^{\prime}$ with $r+1$ components. Since $n=k,|\rho|=$ the number of classes of neighbor lines through the point 0 . Then $|\rho|=r+1=\left|\pi^{\prime}\right|$, and there exists a one-to-one correspondence: $\rho \leftrightarrow \pi^{\prime}, R \leftrightarrow R^{\prime}$. We set $R^{e}=T+R^{\prime}$ for each $R \in \rho . o(T)=r^{2 n}, o\left(R^{e}\right)=r^{2 n+1}$, and (by Lemma 3.1), $o\left({ }^{n-1} T+R\right)=r^{2 n-1}$. Since ${ }^{n-1} T+R \subset T \subset R^{e}$, $R^{e} /\left({ }^{n-1} T+R\right)$ has a congruence, one of whose components is $T /\left({ }^{n-1} T+R\right)=$ $\left({ }^{k} T+R\right) /\left({ }^{k-1} T+R\right)$. The remaining components, we denote as in (3.6). If $k=n$ is 1 , we set $\pi^{e}=\{R[\alpha]\}$.

If $k>1$, we must continue. The construction is now identical for $\pi^{\#}$ and $\pi^{e}$. In order to continue, we are forced to assume that the $H$-plane $\mathfrak{U}$ is strongly $n$ uniform. Suppose the construction has reached the stage in which groups $R\left[\alpha_{1}, \ldots, \alpha_{l}\right]$ have been defined for all $\left(\alpha_{1}, \ldots, \alpha_{l}\right)$ with $l \leq i$ and $0 \leq \alpha_{m}<r$. for all $m$. We take $i$ to be a fixed positive integer $<k$. All groups $R\left[a_{1}, \ldots, \alpha_{l}\right]$ are assumed to be contained in $R^{\#}$ or $R^{e}$ as appropriate. In the following, interpret $R\left[a_{1}, \cdots, \alpha_{i-1}\right]$ to be either $R^{\#}$ or $R^{e}$ as appropriate, when $i=1$. Finally, suppose that if $\left(a_{1}, \cdots, a_{k-1}\right)$ has $\alpha_{l}=0$ for all $l \geq i$, that then

$$
R\left[\alpha_{1}, \cdots, \alpha_{i-1}\right] /\left(R\left(\alpha_{1}, \cdots, \alpha_{k-1}\right)+{ }^{k-i} T\right)
$$

has a congruence whose components are

$$
\left(R\left(\alpha_{1}, \ldots, \alpha_{k-1}\right)+{ }^{k+1-i} T\right) /\left(R\left(\alpha_{1}, \ldots, \alpha_{k-1}\right)+{ }^{k-i} T\right)
$$

and

$$
R\left[\alpha_{1}, \cdots, \alpha_{i-1}, \beta\right] /\left(R\left(\alpha_{1}, \cdots, \alpha_{k-1}\right)+{ }^{k-i} T\right), \quad 0 \leq \beta<r
$$


Observe that when $i=1,(3.8)$ and (3.9) are identical to (3.5) and (3.6) respectively. Next we examine

$$
R\left[\alpha_{1}, \cdots, \alpha_{i}\right] /\left(R\left(\alpha_{1}, \cdots, \alpha_{k-1}\right)+{ }^{k-i-1} T\right)
$$

where $0 \leq \alpha_{l}<r$ for $l \leq i$ and $\alpha_{l}=0$ for $l>i$. First, we give a brief argument to show that the notation in (3.10) does in fact represent a group. We have

$$
R\left(\alpha_{1}, \cdots, \alpha_{k-1}\right)+{ }^{k-i-1} T \subset R\left(\alpha_{1}, \ldots, \alpha_{k-1}\right)+{ }^{k-i} T
$$

which, by (3.4) and Corollary 2.6, is equal to $R\left(\alpha_{1}, \ldots, \alpha_{i-1}, 0, \ldots, 0\right)+{ }^{k-i} T$ which, by (3.9), is contained in $R\left[\alpha_{1}, \ldots, \alpha_{i}\right]$.

If we are working inside $T^{e}$, it is clear that the quotient in (3.10) is elementary abelian. If not, then we have $p \cdot R\left[\alpha_{1}, \cdots, \alpha_{i}\right] \subset p \cdot R^{\#}={ }^{n+1-k} T \cap R$ which, by (3.4), is contained in $R\left(\alpha_{1}, \ldots, \alpha_{k-1}\right)$. Again the quotient (3.10) is elementary abelian. The order of this quotient is $r$ times the common order of the groups at (3.8) and (3.9). By Lemma 3.1, the group in (3.8) has order $r$. Then the groups at (3.10) are all elementary abelian of order $r^{2}$, hence all have congruences with one component equal to

$$
\left(R\left(\alpha_{1}, \cdots, \alpha_{k-1}\right)+{ }^{k-i} T\right) /\left(R\left(\alpha_{1}, \ldots, \alpha_{k-1}\right)+{ }^{k-i-1} T\right) .
$$

The remaining components, we denote by

$$
R\left[\alpha_{1}, \ldots, \alpha_{i}, \beta\right] /\left(R\left(\alpha_{1}, \ldots, \alpha_{k-1}\right)+{ }^{k-i-1} T\right), \quad 0 \leq \beta<r .
$$

We set $\pi^{\#}$ or $\pi^{e}$, as appropriate, equal to

$$
\left\{R\left[\alpha_{1}, \cdots, \alpha_{k}\right]: R \in \rho, 0 \leq \alpha_{l}<r \text { for all } l\right\} .
$$

Recall that the groups listed in (3.9) all have order $r$. Setting $i=k$ in (3.9), we see that the components of $\pi^{\#}$ and $\pi^{e}$ all have order $r$ times the common order $r^{n}$ of the components of $\pi$. For future reference, we state

Lemma 3.2. The components of $\pi^{\#}$ and $\pi^{e}$ all bave order $r^{n+1}$.

Definition 3.1. $J\left(T^{e}, \pi^{e}\right)$ is called a type 1 extension of $J(T, \pi) ; J\left(T^{\#}, \pi^{\sharp}\right)$ is called a type 2 extension of $J(T, \pi)$.

The work of $\$ 3$ yields

Lemma 3.3. Let $\mathfrak{X}=J(T, \pi)$ be an n-uniform translation $H$-plane with trans. lation group isomorpbic to $T$. If $\mathfrak{A}$ is either vertical or strongly $n$-uniform, then $\mathfrak{A}$ bas a type 2 extension. If $\mathfrak{Q}$ is both borizontal and strongly n-uniform, then $\mathfrak{U}$ bas a type 1 extension.

In the next two sections, we shall prove (see Theorem 4.1(b) and Corollary 4.2) that all extensions of both types (which arise from $H$-planes $\mathscr{A}$ satisfying the 
requirements of Lemma 3.3) are strongly $(n+1)$-uniform translation $H$-planes.

We now summarize the two types of constructions. Let $\mathscr{U}=J(T, \pi)$ be a given $n$-uniform translation $H$-plane, $T$ abelian and represented as in Theorem 1.8. Define $T^{\#}$ by (3.2); and if $T$ is elementary abelian, define $T^{e}$ by (3.3). Let $\rho$ be a subset of $\pi$ such that, for each $S \in \pi$, there is a unique $R \in \rho$ satisfying $S \cap R \supset^{n+1-k} T \cap S$. For $R \in \rho$, let

$$
\left\{R\left(a_{1}, \cdots, a_{k-1}\right): 0 \leq \alpha_{i}<r \text { for all } \alpha_{i}\right\}
$$

denote the components of $\pi$ which intersect $R$ in at least $r^{n+1-k}$ points, $R(0, \cdots, 0)=R$. Condition (3.4) must be satisfied. Set $R^{\#}=\left\{t \in T^{\#}: p t \in R\right\}$. Let $\pi^{\prime}$ be a congruence for $C_{0}$ with $r+1$ components $\left(C_{0}\right.$ defined in 3.3). Take a one-to-one correspondence: $\rho \leftrightarrow \pi^{\prime}, R \leftrightarrow R^{\prime}$. Set $R^{e}=T+R^{\prime}$. For $i=1$, $2, \cdots, k$, choose congruences for the groups at (3.7) whose components are given by (3.8) and (3.9). Note that $\alpha_{l}=0$ for all $l \geq i$ in (3.7), (3.8), and (3.9). Interpret the numerator $R\left[a_{1}, \cdots, \alpha_{i-1}\right]$ in (3.7) to be $R^{\#}$ or $R^{e}$, as appropriate, when $i=1$. Then $\pi^{\#}$ or $\pi^{e}$, as appropriate, is the set of subgroups (3.11). Then $J\left(T^{e}, \pi^{e}\right)$ is a type 1 extension of $\mathfrak{U} ; J\left(T^{\#}, \pi^{\sharp}\right)$ is a type 2 extension of $\mathfrak{U}$.

4. The constructibility of all strongly $n$-uniform translation $H$-planes. The goal of $\$ 4$ is to prove part (a) of the following theorem.

Theorem 4.1. (a) Let $B=J\left(T^{*}, \pi^{*}\right)$ be a strongly $(n+1)$-uniform translation $H$-plane, $n \geq 1$, represented so that $T^{*}$ is abelian. Denote ${ }^{n}\left(T^{*}\right)$ by $T$, ${ }^{n}\left(\pi^{*}\right)$, by $\pi$. Then $B$ is a type 1 or a type 2 extension of $J(T, \pi)$ according as $T^{*}$ is or is not elementary abelian.

(b) Conversely, let $\mathfrak{A}=J(T, \pi)$ be any strongly $n$-uniform translation $H$ plane represented with abelian $T$. Then all type 2 extensions of $\mathscr{U}$ and (if $T$ is elementary abelian) all type 1 extensions of $\mathfrak{U}$ are strongly $(n+1)$-uniform trans. lation H-planes.

Proof of (a). If $T^{*}$ is not elementary abelian, Theorem 1.8 assures the existence of subgroups $E_{i}$ such that

$$
T^{*}=E_{1} \oplus \cdots \oplus E_{k}, \quad T=p E_{1} \oplus E_{2} \oplus \cdots \oplus E_{k},
$$

and the $E_{i}$ satisfy all conditions listed for the $C_{i}$ in Theorem 1.8. Then (3.1) and (3.2) are satisfied if we set $T^{\#}=T^{*}, C_{i}=D_{i}=E_{i+1}$ for $i<k, D_{k}=E_{1}$, and $C_{k}=p \cdot D_{k}$. If $T^{*}$ is elementary abelian, Theorem 1.8 implies the existence of subgroups $C_{i}$, each a direct sum of $2 x$ subgroups of order $p$, such that $T^{*}=$ $C_{0} \oplus \cdots \oplus C_{k}$ and $T=C_{1} \oplus \cdots \oplus C_{k}$. We set $T^{e}=T^{*}$ so that (3.1) and (3.3) are satisfied.

At this point, to complete the proof of (a), it suffices to demonstrate that 
congruences may be so chosen that one can obtain a $\pi^{\#}$ or $\pi^{e}$ equal to $\pi^{*}$. We observe that, by Proposition 2.9, ${ }^{n} B=J(T, \pi)$ is a strongly $n$-uniform translation $H$-plane. We pick $\rho$ and label the components of $\pi$ as in \$3. In particular, (3.4) must be satisfied. By Proposition 1.1(11), each $R\left(a_{1}, \ldots, a_{k-1}\right) \in \pi$ is contained in $r$ components of $\pi^{*}$. We denote these $r$ components by $R\left\langle a_{1}, \ldots, a_{k-1}, \beta\right\rangle$, $0 \leq \beta<r$. Let $i$ be a positive integer $\leq k$, and let $\left(\alpha_{1}, \ldots, \alpha_{i}\right)$ be any ordered $i$-tuple whose elements $\alpha_{l}$ are nonnegative integers $<r$. We set

$$
R\left[\alpha_{1}, \ldots, \alpha_{i}\right]=R\left\langle\alpha_{1}, \cdots, \alpha_{k}\right\rangle+{ }^{k-i} T,
$$

taking $\alpha_{l}=0$ for $l>i$.

We now obtain an identity which will prove useful later. Since ${ }^{n} B$ is strongly $n$-uniform, (3.4) and Corollary 2.6 imply that

$$
R\left(\alpha_{1}, \ldots, \alpha_{k-1}\right) \subset^{k-i} T+R\left(\beta_{1}, \ldots, \beta_{k-1}\right)
$$

whenever $\alpha_{l}=\beta_{l}$ for all $l<i$. By symmetry,

$$
{ }^{k-i} T+R\left(\alpha_{1}, \cdots, \alpha_{k-1}\right)={ }^{k-i} T+R\left(\beta_{1}, \cdots, \beta_{k-1}\right)
$$

when $\alpha_{l}=\beta_{l}$ for all $l<i$.

Next, we examine the first set of congruences (components given by (3.5), (3.6) and (4.1)) needed for the construction of $\pi^{e}$. We define $R^{e}$ to be

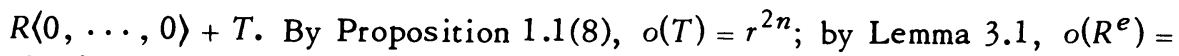
$r^{2 n+1}$. Since $T \subset R^{e} \subset C_{0} \oplus T$ are all elementary abelian, $R^{e}=R^{\prime}+T$ for some $R^{\prime} \subset C_{0}$ with $o\left(R^{\prime}\right)=r$. Let $\pi^{\prime}=\left\{R^{\prime}: R \in \rho\right\}$. Assume $\left|R^{\prime} \cap S^{\prime}\right|>1, R \in \rho, S \in \rho$. Then $R^{e} \cap S^{e}$ contains a point not in $T$. Then $R\langle 0, \ldots, 0\rangle-T$ contains a point neighbor to a point of $S\langle 0, \ldots, 0\rangle$; hence $R\langle 0, \ldots, 0\rangle$ and $S\langle 0, \ldots, 0\rangle$ are intersecting neighbor lines. Then $R$ and $S$ are neighbor lines in $\rho$. By Proposition 1.1(3), $o(S \cap R) \geq r$. Since $k=n$, the choice of $\rho$ implies that $R=S$. Thus distinct components of $\pi^{\prime}$ have trivial intersections; since $|\rho|=r+1, \pi^{\prime}$ is a congruence for $C_{0}$.

It follows from (4.1) and (4.2) that ${ }^{k-1} T+R \subset R[a]$. Since $R\langle a, 0, \ldots, 0\rangle$ and $R\langle 0, \ldots, 0\rangle$ intersect in more than one point, they are neighbor lines; hence by Corollary 2.6, $R\langle\alpha, 0, \ldots, 0\rangle \subset R\langle 0, \ldots, 0\rangle+T=R^{e}$. Then $R[a] \subset R^{e}$. Since $\mathscr{B}$ and $n \mathscr{B}$ have the same invariant $r$, Lemma 3.1 implies that all the groups at (3.5) and (3.6) have order $r$ and that the order of $R^{e} /\left({ }^{k-1} T+R\right)$ is $r^{2}$. Since ${ }^{k} T+R=T+R=T$,

$$
\left({ }^{k} T+R\right) \cap R[\alpha]=\left(R(\alpha, 0, \cdots, 0)+{ }^{k-1} T\right)
$$

which, by (3.4) and Corollary 2.8(a), is equal to $R+{ }^{k-1} T$. We will establish later in the proof that 


$$
R[\alpha] \cap R[\beta]={ }^{k-1} T+R
$$

when $a \neq \beta$. Then the groups at (3.5) and (3.6) for a given $R$ constitute a congruence for $R^{e} /\left({ }^{k-1} T+R\right)$.

Next, we demonstrate that (4.1), (3.5) and (3.6) will also yield congruences for the groups $R^{\#} /\left({ }^{k-1} T+R\right), R^{\#}$ denoting $\left\{t \in T^{\#}: p t \in R\right\}$. By Theorem 1.8, we have $p \cdot R[\alpha]=p \cdot R\langle\alpha, 0, \ldots, 0\rangle \subset R(\alpha, 0, \ldots, 0) \cap{ }^{n+1-k} T$ which, by (3.4), is contained in $R(\alpha, 0, \ldots, 0) \cap R(0, \ldots, 0) \subset R$. Then $R[\alpha] \subset R^{\#}$. It is easily seen that ${ }^{k} T+R \subset R^{\#}$, and it was proved in $\$ 3$ that the order of $R^{\#}(k-1 T+R)$ is $r^{2}$. The remainder of the argument needed to prove that the groups at (3.5) and (3.6) yield congruences for the $R^{\#} /\left({ }^{k-1} T+R\right)$ is identical with the argument of the preceding paragraph.

Finally, we must show that, if we use (4.1) as a definition of the $R\left[\alpha_{1}, \ldots, \alpha_{i}\right]$, then the groups at (3.8) and (3.9) also yield congruences for the groups at (3.7) when $1<i \leq k$. Then assume $i>1$. By (4.1) and (4.2), we have $R\left(\alpha_{1}, \cdots, \alpha_{k-1}\right)+{ }^{k-i} T \subset R\left[\alpha_{1}, \cdots, \alpha_{i}\right]$. By (4.1), (3.4), Proposition 1.1(4), and Corollary 2.6, we have $R\left[\alpha_{1}, \ldots, \alpha_{i}\right] \subset R\left[\alpha_{1}, \ldots, \alpha_{i-1}\right]$. The other necessary inclusions clearly hold. By Lemma 3.1, the groups at (3.8) and (3.9) all have order $r$, and the groups at (3.7) have order $r^{2}$.

Next we show that the groups at (3.8) and (3.9) have trivial intersections, hence that they provide congruences for the groups at (3.7). In the process, we will prove (4.3); the proof of Theorem 4.1(a) will then be complete, since (4.1) and (3.11) imply that $\pi^{*}=\pi^{\#}$ or $\pi^{e}$ as appropriate. Let $i$ be some integer with $1 \leq i \leq k_{\text {. Assume that }} \alpha_{l}=\beta_{l}$ for all $l<i$ and $\alpha_{i} \neq \beta_{i}$. By (3.4) and Proposition $1.1(4)$,

$$
o\left(R\left\langle\alpha_{1}, \cdots, \alpha_{k}\right\rangle \cap R\left\langle\beta_{1}, \cdots, \beta_{k}\right\rangle\right)=r^{n-k+i} .
$$

Then Lemma 2.5 implies that

$$
R\left[\alpha_{1}, \cdots, \alpha_{i}\right] \cap R\left[\beta_{1}, \cdots, \beta_{i}\right] \subset T .
$$

This inclusion, together with (4.1) and (4.2), implies

$$
\begin{aligned}
R\left[\alpha_{1},\right. & \left.\ldots, \alpha_{i}\right] \cap R\left[\beta_{1}, \ldots, \beta_{i}\right] \\
& =\left(R\left(\alpha_{1}, \ldots, \alpha_{k-1}\right)+{ }^{k-i} T\right) \cap\left(R\left(\beta_{1}, \ldots, \beta_{k-1}\right)+{ }^{k-i} T\right) \\
& =R\left(\alpha_{1}, \ldots, \alpha_{k-1}\right)+{ }^{k-i} T
\end{aligned}
$$

where the $\alpha_{l}$ are arbitrary for $l \geq i$. Then, for a given group at (3.7), each two distinct components at (3.9) have a trivial intersection. As a special case, we have (4.3). Since $R\left(a_{1}, \ldots, \alpha_{k-1}\right)+{ }^{k+1-i} T \subset T$, (4.1) and (4.2) imply 


$$
\begin{aligned}
\left(R \left(\alpha_{1},\right.\right. & \left.\left.\cdots, \alpha_{k-1}\right)+{ }^{k+1-i} T\right) \cap R\left[\alpha_{1}, \ldots, \alpha_{i}\right] \\
& =\left(R\left(\alpha_{1}, \ldots, \alpha_{k-1}\right)+{ }^{k+1-i} T\right) \cap\left(R\left(\alpha_{1}, \ldots, \alpha_{k-1}\right)+{ }^{k-i} T\right) \\
& =R\left(\alpha_{1}, \ldots, \alpha_{k-1}\right)+{ }^{k-i} T .
\end{aligned}
$$

The proof of Theorem 4.1(a) is complete.

Before proceeding to a proof of Theorem 4.1(b), we first give an alternate proof of (a) in the special case $T=p T^{*}$, replacing the assumption of strong $(n+1)$-uniformity for $B$ by the weaker assumption of $(n+1)$-uniformity. Since 1 uniform $H$-planes are all strongly 1 -uniform, We may then apply Remark 1.9, Proposition 1.10(a), and Theorem 4.1(b) to obtain the following corollary.

Corollary 4.2. Vertical $n$-uniform translation $H$-planes are strongly $n$-uniform.

Proof. It remains to prove that the conclusion of Theorem 4.1(a) holds when one replaces the hypothesis of strong $(n+1)$-uniformity for $B$ by the hypothesis of $(n+1)$-uniformity and the additional assumption $k=1$. Since $k=1$, there is only one round of congruences to find. For each $R \in \pi$, let $R[\alpha], 0 \leq \alpha<r$, denote the $r$ lines of $\pi^{*}$ which contain $R$. The proof will be complete if we can demonstrate that

$$
\left\{\left({ }^{1} T+R\right) / R\right\} \cup\{R[\alpha] / R: 0 \leq \alpha<r\}
$$

is a congruence for $R^{\#} / R$. We have ${ }^{1} T+R, R[\alpha] \subset R^{\#}$ for all $\alpha$. Also, $R[\alpha] \cap$ $R[\beta]=R$ if $\alpha \neq \beta$; and since ${ }^{1} T+R \subset T,\left({ }^{1} T+R\right) \cap R[\alpha]=R$. Then the $r+1$ components, each of order $r$, constitute a congruence for $R^{\#} / R$.

\section{The converse.}

Proof of Theorem 4.1(b). Our assumption is that $\mathcal{U}=J(T, \pi)$ is a strongly $n$ uniform translation $H$-plane represented with abelian $T$. We will verify that $(\mathbb{S}=$ $J\left(T^{\#}, \pi^{\sharp}\right)$ and (if $T$ is elementary abelian) $\mathfrak{D}=J\left(T^{e}, \pi^{e}\right)$ satisfy conditions (a)(g) of Theorem 1.5. Since

$$
p T^{\#}={ }^{n+1-k} T \subset \bigcup_{R \in \rho} R, \quad T^{\#}=\bigcup_{R \in \rho} R^{\#} .
$$

Since the groups at (3.5) and (3.6) constitute a congruence of $R^{\#} /\left({ }^{k-1} T+R\right)$,

$$
R^{\#}-\left({ }^{k} T+R\right) \subset \underset{0 \leq \alpha<r}{\bigcup} R[\alpha]
$$

and similarly

$$
R\left[\alpha_{1}, \ldots, \alpha_{i-1}\right]-\left(R\left(\alpha_{1}, \ldots, \alpha_{k-1}\right)+{ }^{k+1-i} T\right) \subset \bigcup_{0 \leq \beta<r} R\left[\alpha_{1}, \ldots, \alpha_{i-1}, \beta\right]
$$

for $2 \leq i \leq k$. Then $T^{\#}-T \subset S=\bigcup_{R}\left[\alpha_{1}, \ldots, \alpha_{k}\right\}$, the union being taken over all components of $\pi^{\#}$. But $T \subset \bigcup_{Q \epsilon \pi} Q$; then by examining (3.9) with $i=k$, we see that $T \subset S$; hence $T^{\#} \subset S$. Clearly $T^{e}=\bigcup_{R \in \rho} R^{e}$, and $R^{e}-T \subset \bigcup_{0 \leq \alpha<r} R[\alpha]$. 
We may now proceed as before to see that $T^{e} \subset S$. We have proved that $(S$ and $D$ both satisfy Theorem $1.5(\mathrm{a})$.

If $P, Q \in \pi^{\sharp}$ or $P, Q \in \pi^{e}$ and if $P \cap Q=0$, then Lemma 3.2 implies $o(P+Q)$ $=r^{2 n+2}=o\left(T^{\#}\right)$ or $o\left(T^{e}\right)$, respectively. Then $(\mathcal{S}$ and $D$ both satisy (b).

In order to verify that $(S$ and $D$ also satisfy conditions $(\mathrm{c})-(\mathrm{g})$ of Theorem 1.5 , it is necessary to determine the intersections of all pairs of components of $\pi^{\#}$ and $\pi^{e}$. We proceed to make this determination. From (3.1), Theorem 1.8, and the definition of $R^{\#}$, we have $R^{\#} \cap T=\{t \in T: p t \in R\}=\left\{t \in T: p t \epsilon^{n-k} T \cap R\right\}$. Then Theorem 1.8 and Lemma 3.1 imply that $o\left(R^{\#} \cap T\right)=r^{2 k} \cdot r^{n-k}=r^{n+k}$, $\left.o^{(k} T+R\right)=r^{n+k}$. Since ${ }^{k} T+R \subset R^{\sharp} \cap T$, one has

$$
{ }^{k} T+R=R^{\sharp} \cap T \text {. }
$$

Since we are assuming that $\mathfrak{A}$ is strongly $n$-uniform, (3.4) implies (4.2) as before. Then

$$
{ }^{k-1} T+R={ }^{k-1} T+R\left(\alpha_{1}, \cdots, \alpha_{k-1}\right)
$$

for all $\alpha_{1}, \ldots, \alpha_{k-1}$. Then ${ }^{k} T+R={ }^{k} T+R\left(\alpha_{1}, \ldots, \alpha_{k-1}\right)$, so (5.1) implies

$$
{ }^{k} T+R\left(\alpha_{1}, \cdots, \alpha_{k-1}\right)=R^{H} \cap T \text {. }
$$

In the construction of $D, k=n$ and $R^{e} \cap T=T$. Then identities (5.1) and (5.3) are also valid if one replaces $R^{\#}$ by $R^{e}$. Henceforth, we write $R^{x}$ whenever we wish to indicate that a statement is valid both for $R^{\#}$ and $R^{e}$. Similarly, we will sometimes write $\pi^{x}$ and $T^{x}$.

Assume $t \in T \cap R\left[\alpha_{1}, \ldots, \alpha_{k}\right]$. Then $t \in R\left[\alpha_{1}, \ldots, \alpha_{k-1}\right] \subset \ldots \subset R\left[\alpha_{1}\right] \subset R^{x}$. By (5.1), $t \in R\left[\alpha_{1}\right] \cap\left(R^{x} \cap T\right)=R\left[\alpha_{1}\right] \cap\left({ }^{k} T+R\right)$. Since $R\left[\alpha_{1}\right] /\left({ }^{k-1} T+R\right)$ and $\left({ }^{k} T+R\right) /\left({ }^{k-1} T+R\right)$ are components of a congruence, we have $t \in{ }^{k-1} T+R$. By (5.2), $t \epsilon^{k-1} T+R\left(\alpha_{1}, \cdots, \alpha_{k-1}\right)$. Assume $t \epsilon^{k-i} T+R\left(\alpha_{1}, \cdots, \alpha_{k-1}\right)$ for some $i<k$. Then

$$
t \in R\left[\alpha_{1}, \ldots, \alpha_{i+1}\right] \cap\left(R\left(\alpha_{1}, \ldots, \alpha_{k-1}\right)+{ }^{k-i} T\right)
$$

By examining the appropriate congruence, we see that $t \in R\left(\alpha_{1}, \ldots, \alpha_{k-1}\right)+$ $k^{-i-1} T$. By induction, $t \in R\left(\alpha_{1}, \cdots, \alpha_{k-1}\right)$. Then

$$
T \cap R\left[\alpha_{1}, \cdots, \alpha_{k}\right] \subset R\left(a_{1}, \cdots, \alpha_{k-1}\right) .
$$

The reverse inclusion follows by setting $i=k$ in (3.9). Then

$$
T \cap R\left[\alpha_{1}, \cdots, \alpha_{k}\right]=R\left(a_{1}, \ldots, \alpha_{k-1}\right) .
$$

Let $t \in R\left[\alpha_{1}, \cdots, \alpha_{k}\right] \cap R\left[\beta_{1}, \cdots, \beta_{k}\right]$, not all $\alpha_{i}=\beta_{i}$. Let $i$ be the smallest positive integer such that $\alpha_{i} \neq \beta_{i}$. Then by (3.9), 


$$
t \in R\left[\alpha_{1}, \ldots, \alpha_{i}\right] \cap R\left[\beta_{1}, \cdots, \beta_{i}\right]=R\left(\alpha_{1}, \ldots, \alpha_{k-1}\right)+{ }^{k-i} T .
$$

It follows from (5.4) that

$$
\begin{aligned}
R\left(\alpha_{1}, \ldots, \alpha_{k-1}\right) & \subset\left(R\left(\alpha_{1}, \ldots, \alpha_{k-1}\right)+{ }^{k-i} T\right) \cap R\left[\alpha_{1}, \ldots, \alpha_{k}\right] \\
& \subset T \cap R\left[\alpha_{1}, \ldots, \alpha_{k}\right]=R\left(\alpha_{1}, \ldots, \alpha_{k-1}\right) .
\end{aligned}
$$

Thus $R\left(\alpha_{1}, \ldots, \alpha_{k-1}\right)=\left(R\left(\alpha_{1}, \ldots, \alpha_{k-1}\right)+{ }^{k-i} T\right) \cap R\left[a_{1}, \ldots, \alpha_{k}\right]$; hence $t \in R\left(\alpha_{1}, \cdots, \alpha_{k-1}\right)$. It follows that

(5.5) $R\left[\alpha_{1}, \ldots, \alpha_{k}\right] \cap R\left[\beta_{1}, \cdots, \beta_{k}\right]=R\left(\alpha_{1}, \ldots, \alpha_{k-1}\right) \cap R\left(\beta_{1}, \ldots, \beta_{k-1}\right)$

unless $\alpha_{i}=\beta_{i}$ for all $i$.

Next, suppose $R, S \in \rho, R \neq S$. Then by Proposition 1.1(3), (9), $R \cap S \subset$ ${ }^{n-k} T \cap R$. Let $t \in R\left[\alpha_{1}, \cdots, \alpha_{k}\right] \cap S\left[\beta_{1}, \cdots, \beta_{k}\right]$. Then $t \in R^{x} \cap S^{x}$. If $x=\#$, we have $p t \in R \cap S \subset{ }^{n-k} T$; hence $t \in T$. If $x=e$, we have $t \in R^{e} \cap S^{e}=T$. Then, in both cases, (5.4) implies that $R\left[\alpha_{1}, \cdots, \alpha_{k}\right] \cap s\left[\beta_{1}, \cdots, \beta_{k}\right]$ is contained in (hence equal to) $R\left(\alpha_{1}, \cdots, \alpha_{k-1}\right) \cap S\left(\beta_{1}, \cdots, \beta_{k-1}\right)$ when $R \neq S$. This result, together with $(5.5)$, yields

$$
R\left[\alpha_{1}, \ldots, \alpha_{k}\right] \cap S\left[\beta_{1}, \ldots, \beta_{k}\right]=R\left(\alpha_{1}, \ldots, \alpha_{k-1}\right) \cap S\left(\beta_{1}, \ldots, \beta_{k-1}\right)
$$

unless $R=S$ and $\alpha_{i}=\beta_{i}$ for all $i$.

Since $\mathfrak{A}$ must satisfy Theorem $1.5(\mathrm{c}),(5.6)$ implies that $\mathcal{S}$ and $\mathcal{I}$ do also. By (5.4) and (5.6), the group $T$ is equal to the set $N$ of Theorem 1.5(d). Hence, both $(5$ and $D$ satisfy condition (d). It follows from (5.4) and Lemma 3.2 that (e) is satisfied. Let $P, Q \in \pi^{x}$ such that $P \cap Q=\{0\}$. Then, by condition (b), $T^{x}=$ $P+Q=(T+P)+(T+Q)$. Then

$$
r^{2 n+2}=o\left(T^{x}\right)=o(T+P) \cdot o(T+Q) / o((T+P) \cap(T+Q)) .
$$

By (5.4) and Lemma 3.2,o $(T+P)=r^{2 n} \cdot r^{n+1} / o(T \cap P)=r^{2 n+1}$. Then (5.7) implies that $o((T+P) \cap(T+Q))=r^{2 n}=o(T)$. Since $T \subset(T+P) \cap(T+Q)$, condition (f) is satisfied.

Clearly, $T+R\left[\alpha_{1}, \cdots, \alpha_{k}\right] \subset T+R^{x}$; we wish to prove

$$
T+R\left[\alpha_{1}, \cdots, \alpha_{k}\right]=T+R^{x} \text {. }
$$

By $(5.1), o\left(T+R^{x}\right)=o(T) \cdot o\left(R^{x}\right) / o\left({ }^{k} T+R\right)=r^{2 n} \cdot o\left(R^{x} /\left({ }^{x} T+R\right)\right)=r^{2 n+1}, \mathrm{By}$ (5.4),

$$
o\left(T+R\left[\alpha_{1}, \ldots, \alpha_{k}\right]\right)=o(T) \cdot o\left(R\left[\alpha_{1}, \ldots, \alpha_{k}\right]\right) / o\left(R\left(a_{1}, \ldots, \alpha_{k-1}\right)\right) .
$$

By Lemma 3.2, this order is also $r^{2 n+1}$; and (5.8) is established.

Assume $o\left(R\left[\alpha_{1}, \ldots, \alpha_{k}\right] \cap S\left[\beta_{1}, \ldots, \beta_{k}\right]\right)>1$. If we can prove that

$$
T+R^{x}=T+S^{x},
$$


the validity of condition (g) will follow from (5.8). We handle first the case $x=\#$. Let $t \in R^{\#}$. By Theorem 1.8, pt $\in^{n+1-k} T \cap R$. Then $p t(\simeq j) 0$ in $\mathcal{U}$ for some integer $j \geq k-1$. By (5.6), $R\left(\alpha_{1}, \ldots, \alpha_{k-1}\right)$ and $S\left(\beta_{1}, \ldots, \beta_{k-1}\right)$ are neighbor

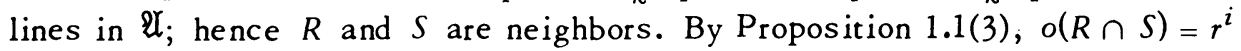
for some positive integer $i$. If $i+j<n$, Lemma 2.5 implies that $p t \epsilon^{n-i-j} T+S$ $C^{n-k} T+S$. If $i+j \geq n$, Proposition 1.1(9) implies that $p t \in S \subset{ }^{n-k} T+S$. Then $p t=x+y$ for some $x \in S$, some $y \epsilon^{n-k} T$. Since $x \epsilon^{n+1-k} T \cap S$, there exists an element $u \in S^{\#}$ with $p u=x$; there is also an element $v \in T$ with $p v=y$. Let $w=$ $t-u-v$. Then $p t=p u+p v+p w=x+y+p w=p t+p w$. Then $p w=0$, so $w \in{ }^{k} T$. Then $t=u+v+w \in S^{\#}+T$. Then $R^{\#} \subset S^{\#}+T$. By symmetry, $S^{\#} \subset R^{\#}+T$, and (5.9) follows in the case $x=\#$. Next, let $x=e$. Again the assumption and (5.6) imply $|R \cap S|>1$. But now we have $k=n$ which forces $R=S$, and (5.9) follows trivially. This completes the proof that condition $(\mathrm{g})$ holds. Then Theorems 1.5 and 1.6 imply that $\mathcal{E}$ and $\mathscr{D}$ are both translation $H$-planes.

By assumption, $\mathfrak{X}=J(T, \pi)$ is $n$-uniform; and by (5.4) every line of $\mathscr{U}$ is the restriction of $r$ lines of $\sqrt{S}$ or $\mathscr{P}$. Since $\sqrt{ }$ and $\mathscr{D}$ are translation $H$-planes, both of the above properties are satisfied by the neighborhood of each point. Then both $\mathbb{S}$ and 9 are $(n+1)$-uniform with invariant $r$.

To prove strong $(n+1)$-uniformity, we need the following result.

Lemma 5.1. If $o\left(R\left[\alpha_{1}, \ldots, \alpha_{k}\right] \cap s\left[\beta_{1}, \ldots, \beta_{k}\right]\right)=r^{j}$, then

$$
R\left[\alpha_{1}, \ldots, \alpha_{k}\right]+S\left[\beta_{1}, \ldots, \beta_{k}\right]={ }^{n+1-j}\left(T^{x}\right)+R\left[\alpha_{1}, \ldots, \alpha_{k}\right] .
$$

Proof. If $R=S$ and $\alpha_{i}=\beta_{i}$ for all $i$, then $j=n+1$, and the lemma's conclusion is clear. Assume $R=S$ and that there is a smallest integer $i$ for which $\alpha_{i} \neq \beta_{i}$. By examining the appropriate congruence of $\$ 3$, we see that $R^{x}$ (if $i=1$ ) or $R\left[\alpha_{1}, \ldots, \alpha_{i-1}\right]$ (in case $i \neq 1$ ) is equal to

$$
R\left[\alpha_{1}, \ldots, \alpha_{i}\right]+R\left[\beta_{1}, \ldots, \beta_{i}\right]=R\left[\alpha_{1}, \ldots, \alpha_{i}\right]+{ }^{k+1-i} T+R\left(\alpha_{1}, \ldots, \alpha_{k-1}\right) \text {. }
$$

To see that (5.10) holds for $i=1$, apply (3.4), Lemma 3.1, and Corollary 2.6 to obtain ${ }^{k-1} T+R={ }^{k-1} T+R\left(\alpha_{1}, \ldots, \alpha_{k-1}\right)$, hence ${ }^{k} T+R={ }^{k} T+$ $R\left(\alpha_{1}, \ldots, \alpha_{k-1}\right)$. From (5.10), we obtain

$$
\begin{aligned}
R\left[\alpha_{1}, \ldots, \alpha_{k}\right]+ & R\left[\beta_{1}, \ldots, \beta_{k}\right] \subset R\left[\alpha_{1}, \ldots, \alpha_{i}\right]+R\left[\beta_{1}, \ldots, \beta_{i}\right] \\
& =R\left[\alpha_{1}, \ldots, \alpha_{i}\right]+{ }^{k+1-i} T+R\left(\alpha_{1}, \ldots, \alpha_{k-1}\right) \\
& ={ }^{k+1-i} T+R\left[\alpha_{1}, \ldots, \alpha_{i}\right] .
\end{aligned}
$$

From (5.5), (3.4), and Lemmas 3.1 and 3.2, we obtain

$$
\begin{aligned}
o\left(R \left[\alpha_{1},\right.\right. & \left.\left.\ldots, \alpha_{k}\right]+R\left[\beta_{1}, \ldots, \beta_{k}\right]\right) \\
& =r^{2 n+2} / o\left(R\left(\alpha_{1}, \ldots, \alpha_{k-1}\right) \cap R\left(\beta_{1}, \ldots, \beta_{k-1}\right)\right)=r^{n+k+2-i}
\end{aligned}
$$


By examining the appropriate congruence from $\$ 3$ and applying (5.10) and Lemma 3.1 , we see that $o\left(R^{x}\right)$ or $o\left(R\left[\alpha_{1}, \ldots, \alpha_{i-1}\right]\right)$ is equal to

$$
\begin{aligned}
o\left(R\left[\alpha_{1}, \ldots, \alpha_{i}\right]+{ }^{k+1-i} T+R\left(\alpha_{1}, \ldots, \alpha_{k-1}\right)\right) \\
=r^{2} \cdot o\left(R\left(\alpha_{1}, \ldots, \alpha_{k-1}\right)+{ }^{k-i} T\right)=r^{n+k+2-i} .
\end{aligned}
$$

Then (5.11), (5.12), and (5.13) imply

$$
R\left[\alpha_{1}, \ldots, \alpha_{k}\right]+R\left[\beta_{1}, \ldots, \beta_{k}\right]={ }^{k+1-i} T+R\left[\alpha_{1}, \ldots, \alpha_{i}\right] .
$$

By Lemma 3.1, o $\left.{ }^{k+1-i} T+R\left[\alpha_{1}, \ldots, \alpha_{k}\right]\right)=r^{n+k+2-i}$. Then

$$
R\left[\alpha_{1}, \ldots, \alpha_{k}\right]+R\left[\beta_{1}, \ldots, \beta_{k}\right]={ }^{k+1-i} T+R\left[\alpha_{1}, \ldots, \alpha_{k}\right] .
$$

Let $j$ be as in the statement of Lemma 5.1. Using (5.5), (3.4), and Lemma 3.1, we see that

$$
r^{j}=o\left(R\left(\alpha_{1}, \ldots, \alpha_{k-1}\right) \cap R\left(\beta_{1}, \ldots, \beta_{k-1}\right)\right)=r^{n-k+i} .
$$

Then $j=n-k+i$, hence $n+1-j=k+1-i$. Since each line of $\mathcal{Q}$ is the restriction of exactly $r$ lines of $\left(\mathcal{C}\right.$ or $\mathscr{D},{ }^{l}\left(T^{x}\right)={ }^{l} T$ for $l \leq n$. Then the conclusion of Lemma 5.1 follows from (5.14) in the case that $R=S$.

Henceforth, assume $R \neq S$. We consider first the plane $\mathcal{D}$. In the construction of $\mathfrak{D}, k=n$; so $R \neq S$ implies that $R\left(\alpha_{1}, \ldots, \alpha_{k-1}\right)$ and $S\left(\beta_{1}, \ldots, \beta_{k-1}\right)$ have a trivial intersection. Then (5.6) implies $j=0$, hence by Lemma 3.2,

$$
o\left(R\left[\alpha_{1}, \ldots, \alpha_{k}\right]+S\left[\beta_{1}, \ldots, \beta_{k}\right]\right)=r^{2 n+2} .
$$

Then $R\left[\alpha_{1}, \ldots, \alpha_{k}\right]+S\left[\beta_{1}, \ldots, \beta_{k}\right]={ }^{n+1}\left(T^{e}\right)$, and the conclusion of Lemma 5.1 holds.

Finally, we must prove that the lemma holds in $\sqrt{ }$ when $R \notin S$. It follows from (5.6) and Corollary 2.6 that

$$
s\left(\beta_{1}, \ldots, \beta_{k-1}\right) \subset^{n-j} T+R\left(\alpha_{1}, \ldots, \alpha_{k-1}\right) .
$$

It follows from Lemma 3.1 that

$$
R\left(\alpha_{1}, \ldots, \alpha_{k-1}\right)+S\left(\beta_{1}, \ldots, \beta_{k-1}\right)={ }^{n-j} T+R\left(\alpha_{1}, \ldots, \alpha_{k-1}\right) .
$$

Then

$$
R\left[\alpha_{1}, \ldots, \alpha_{k}\right]+S\left(\beta_{1}, \ldots, \beta_{k-1}\right)={ }^{n-j} T+R\left[\alpha_{1}, \ldots, \alpha_{k}\right] .
$$

Next we compute $o\left(R^{\#}+S^{\#}\right)=o\left(R^{\#}\right)^{2} / o\left(R^{\#} \cap S^{\#}\right)$. Define $\phi$ from $T^{\#}$ to $T^{\#}$ by $\phi(t)=p t$. Then by Theorem 1.8 and Lemma 3.1(a),

$$
o\left(R^{\#}\right)=o(\operatorname{Image} \phi \cap R) \cdot o(\operatorname{Ker} \phi)=o\left(R \cap{ }^{n+1-k} T\right) \cdot r^{2 k}=r^{n+1+k} \text {. }
$$


Since $R^{\#} \cap S^{\#}=\left\{t \in T^{\#}: p t \in R \cap S\right\}$,

$$
o\left(R^{\#} \cap S^{\#}\right)=o(\text { Image } \phi \cap R \cap S) \cdot o(\operatorname{Ker} \phi) \text {. }
$$

Since $R \neq S, j \leq n-k$; and Proposition 1.1(9) implies that $o(R \cap S)=r^{j}$. Then by Proposition 1.1(9), $R \cap S \subset{ }^{j} T \subset{ }^{n+1-k} T=$ Image $\phi$, and $o\left(R^{\#} \cap S^{\sharp}\right)=r^{j+2 k}$. Putting things together, we see that $o\left(R^{\#}+S^{\#}\right)=r^{2 n+2-j}$. Then

$$
R^{\#}+S^{\#}=R\left[\alpha_{1}, \cdots, \alpha_{k}\right]+S\left[\beta_{1}, \cdots, \beta_{k}\right] \text {. }
$$

Now let $t \epsilon^{n+1-j}\left(T^{\sharp}\right)$. Then Theorem 1.8 implies $p t \epsilon^{n+1-j-k} T$, and (5.15) implies

$$
p t \in\left(R\left[\alpha_{1}, \ldots, \alpha_{k}\right]+S\left[\beta_{1}, \ldots, \beta_{k}\right]\right) \cap{ }^{n+1-k} T .
$$

Then there exist $a, b \in T^{\#}$ such that $p t=p a+p b$,

$$
p a \in R\left[\alpha_{1}, \ldots, \alpha_{k}\right] \cap{ }^{n+1-k} T \subset R, \quad p b \in S\left[\beta_{1}, \cdots, \beta_{k}\right] \cap{ }^{n+1-k} T \subset S .
$$

Let $c$ satisfy $t=a+b+c$. Then $p c=0$, so $p(b+c) \in S$. Then $a \in R^{\#}$, and $(b+c) \in S^{\#}$; so $t \in R^{\#}+S^{\#}$. Then (5.16) implies that

$$
n+1-j\left(T^{\#}\right) \subset R\left[\alpha_{1}, \cdots, \alpha_{k}\right]+S\left[\beta_{1}, \cdots, \beta_{k}\right] \text {. }
$$

The conclusion of Lemma 5.1 follows by an easy cardinality argument. This completes the proof of the lemma.

Proof of Theorem 4.1(b) resumed. It remains to prove that $\mathbb{E}$ and 9 satisfy Axiom $S$. Let $P, Q \in \pi^{x}$, and let $r^{i}$ denote $o(P \cap Q), i \leq n$. Let $c \in Q-P$. By Proposition 1.1(9), $c(\simeq j) 0$ for some $j<n+1-i$. If $j \neq 0, c(\simeq j-1) 0$ in $\mathcal{U}$. Since $\mathfrak{A}$ satisfies Axiom S, $c(\simeq i+j-1)(P \cap T)$ in $\mathcal{U}$. Then $c(\simeq i+j) P$ in $(\mathbb{S}$ and $\mathscr{D}$.

Next, we consider the case $j=0$. By Lemma 5.1, $c \epsilon^{n+1-i}\left(T^{x}\right)+P$. Then $c(\sim i)$ P. By Proposition 1.1(8), the number of points $d \in T^{x}$ such that $c(\sim i+1) d$ is $r^{2 n-2 i}$. Lemma 5.1 implies that if $X \in \pi^{x}$ and $o(X \cap Q) \geq r^{i+1}$, then $c(\sim i+1) X$. By Proposition 1.1(4) and (6), there are exactly $r^{n-i}$ points $d$ on $X$ satisfying $c(\sim i+1) d$. The $r^{n-i}$ lines $X \in \pi^{x}$ satisfying $o(X \cap Q) \geq$ $r^{i+1}$ thus must contain all $r^{2 n-2 i}$ points $d$ wi th $c(\sim i+1) d$. Then $c(\not x i+1)$ $P$, so $c(\simeq i) P$. Then $\sqrt{ }$ and $\mathscr{D}$ both satisfy Axiom $S$ in the special case that the lines pass through 0 . Since $\mathcal{C}$ and $\mathscr{D}$ are translation $H$-planes, they must satisfy Axiom $\mathrm{S}$ without restriction.

6. Variability of the $\left.{ }^{(i \mathcal{Q}}\right)_{j}$. In Proposition $1.10(\mathrm{c})$ and Remark 1.11, we obtained limits to the variability of the substructures $\left({ }^{i} \mathfrak{U}\right)_{j}$ of an $n$-uniform translation $H$-plane 2 . In this section, we obtain results which show that the earlier limits are, in some sense, best possible. In other words, the maximum variability in the $\left({ }^{i}()_{j}\right.$ permitted by the earlier results does occur and, in fact, occurs within the 
subclass of strongly $n$-uniform $H$-planes. The results are all heavily dependent upon Theorem 4.1. We begin by proving

Remark 6.1. If $B$ is a strongly $i$-uniform translation $H$-plane and if $\mathcal{U}$ is obtained from $B$ by a finite number of extensions of types 1 and 2 , then $i \mathfrak{U}=\mathbb{B}$. Conversely, let $\mathcal{U}$ be a strongly $(i+j)$-uniform translation $H$-plane, $i \geq 1$. Then OU can be obtained from $i \mathfrak{Q}$ by $j$ extensions of types 1 and 2 .

Proof. Let $\mathfrak{B}$ be a strongly $i$-uniform translation $H$-plane. Let $\mathbb{E}_{k}$ be obtained from $\mathbb{E}_{k-1}$ by a single type 1 or type 2 extension, $1 \leq k \leq j ; \mathbb{E}_{0}=\mathbb{B}_{;} \mathbb{E}_{j}=2$ C. By (5.4), each line of $\mathbb{E}_{k-1}$ is the restriction of exactly $r$ lines of $\mathbb{E}_{k}$ : hence each point of $\mathbb{E}_{k-1}$ is joined to 0 by $r$ times as many lines in $\mathbb{E}_{k}$ as in $\mathbb{F}_{k-1}$. By (5.6), each point of $\mathbb{E}_{k}-\mathbb{E}_{k-1}$ is joined to 0 by exactly 1 line of $\mathbb{E}_{k}$. Then the points of $\mathscr{B}$ are precisely the points of $\mathfrak{U}$ which are joined to 0 by $r^{j}$ or more lines of $\mathcal{U}$. Then $M$ and $i \mathcal{U}$ have the same point set; and (5.4) implies that they have the same line set, same incidence, and same parallel relation.

To establish the converse, let $\mathcal{U}$ be a strongly $(i+j)$-uniform translation $H$. plane with $i \geq 1$. By Theorem 4.1(a) and Proposition 2.9, थ can be obtained by $j$ extensions of some strongly $i$-uniform translation $H$-plane $\$$. By the first conclusion, $B=i$ U.

Remark 6.2. If $i$ and $j$ are any specified positive integers and if $B$ is any strongly $i$-uniform translation $H$-plane, then there exists a strongly $(i+j)$-uniform translation $H$-plane $\mathscr{U}$ such that $i \mathscr{U}=\mathbb{P}$. Suppose the width of $\mathbb{B}$ is $k$. If $k<i$, OU must have width $k$; if $k=i$, $\mathcal{U}$ may be required to have any specified width $b$ in the range $i \leq b \leq i+j$.

Proof. Given $\mathbb{B}$, Lemma 3.3 and Theorem 4.1(b) permit one to make $j$ extensions to obtain $\mathcal{Q}$. By Remark 6.1, ${ }^{i} \mathfrak{U}=\mathfrak{B}$. It also follows from Remark 6.1 that every $\mathfrak{U}$ with the desired properties can be obtained from $\mathfrak{B}$ by $j$ extensions. If $k<i$, Theorems 4.1(a) and 1.8 imply that every extension must be of type 2; hence I must have width $k$. If $k=i$, one may make $(b-i)$ type-1 extensions, followed by $(i+j-b)$ type-2 extensions.

Theorem 6.3. Let $\Re$ and $(5$ be horizontal translation H-planes, both with invariant $r, \mathbb{B}$ being strongly $i$-uniform and $\mathbb{S}$ being strongly $j$-uniform. Then there exists a borizontal strongly $(i+j)$-uniform translation $H$-plane O with invariant

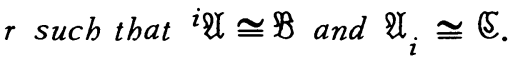

Proof. We prove the corollary by induction on $j$. The induction hypothesis is that under the hypotheses stated in the theorem, one can obtain an $H$-plane $\mathcal{O}$ by $j$ type-1 extensions of $B$ so that $\mathfrak{U}_{i} \cong \mathfrak{S}$. Once the induction proof is complete, the truth of the theorem will follow from Remark 6.1.

Assume first that $j=1$. Let $B=J(T, \pi), T$ abelian. Let $T^{e}=C_{0} \oplus T, C_{0}$ 
being the elementary abelian group of order $r^{2}$. André has proved [1, Satz 9] that the ordinary translation plane $\mathfrak{S} \cong J\left(C_{0}, \pi^{\prime}\right)$ for some congruence $\pi^{\prime}$ of $C_{0}$. We use this $\pi^{\prime}$ to obtain a type-1 extension of $\mathfrak{B}$ to $\mathfrak{U}=J\left(T^{e}, \pi^{e}\right), T^{e}=C_{0} \oplus T$. Since $\mathcal{P}$ is $(i+1)$-uniform, ${ }^{i}\left(T^{e}\right)$ is the set of all points of $T^{e}$ which are neighbor (in $\mathfrak{f}$ ) to 0 . It is clear from (5.6) that ${ }^{i}\left(T^{e}\right)=T$. Then $\left(T^{e}\right)_{i}=\left(C_{0} \oplus T\right) / T$. Let $a$ be the canonical (group) isomorphism from $\left(C_{0} \oplus T\right) / T$ onto $C_{0}$. We consider the image under $\alpha$ of a typical element of $\left(\pi^{e}\right)_{i}$. By (5.8), we have $\left.a\left(\left(R\left[\alpha_{1}, \cdots, \alpha_{i}\right]+T\right) / T\right)=\alpha\left(R^{e}+T\right) / T\right)=\alpha\left(\left(R^{\prime}+T\right) / T\right)=R^{\prime} \in \pi^{\prime}$. It follows from Theorem 1.7, that $\hat{U}_{i}=J\left(\left(C_{0} \oplus T\right) / T,\left(\pi^{e}\right)_{i}\right)$ is isomorphic to $J\left(C_{0}, \pi^{\prime}\right) \cong \mathfrak{E}$. Then the induction hypothesis is satisfied for $j=1$.

Now let $B$ and $\mathscr{D}$ be given horizontal translation $H$-planes, $B$ strongly $i$ uniform, and $\mathscr{D}$ strongly $(j+1)$-uniform, $j \geq 1$. Then $\mathscr{S}$ can be created by a single type- 1 extension from a horizontal strongly $j$-uniform translation $H$-plane $\sqrt{ } 5$. By the induction assumption, one can obtain an $H$-plane $\mathcal{U}$ by $j$ type- 1 extensions of $B$ so that $\mathfrak{U}_{i} \cong \mathfrak{S}$. The induction proof will be complete if we prove the existence of a type-1 extension $\mathbb{E}$ of $\mathscr{U}$ such that $\mathbb{E}_{i} \cong \mathfrak{I}$. Let $\mathcal{U}$ be represented by $J(T, \pi)$, (S by $J(U, \mu)$, both $T$ and $U$ abelian. Since $\mathfrak{S} \cong \mathfrak{U}_{i}=J\left(T_{i}, \pi_{i}\right)$, Theorem 1.7 implies the existence of a (group) isomorphism $\alpha$ from $T /{ }^{i} T$ onto $U$ such that $\alpha\left(\left(R+{ }^{i} T\right) /{ }^{i} T\right)=R^{*} \in \mu$ for each $R \in \pi$. Let $\beta$ be the canonical homomorphism of $T$ onto $T /{ }^{i} T$, and set $\gamma=\alpha \beta$. We summarize in the following diagram:

$$
T \stackrel{\beta}{\longrightarrow} T /{ }^{i} T \stackrel{a}{\longrightarrow} U, \quad \gamma=\alpha \beta \text {. }
$$

We have $\mathscr{D}=J\left(C_{0} \oplus U, \mu^{e}\right)$ where $\mu^{e}$ is a collection of subgroups of $C_{0} \oplus U$. The members of $\mu^{e}$ are denoted by $R^{*}\left[\alpha_{1}, \ldots, \alpha_{j}\right], R^{*} \in \nu \subset \mu, 0 \leq \alpha_{k}<r$ for $1 \leq k \leq j$.

If $\mathbb{E}=J\left(C_{0} \oplus T, \pi^{e}\right)$ is any type-1 extension of $\mathcal{U}$, then Lemma 1.2 implies that ${ }^{i}\left(C_{0}+T\right)={ }^{i} T$. Let $\beta^{\prime}$ be the canonical homomorphism of $C_{0} \oplus T$ onto $\left(C_{0} \oplus T\right) /{ }^{i} T$. Let $\gamma^{\prime}$ be the homomorphism from $C_{0} \oplus T$ onto $C_{0} \oplus U$ induced by $\gamma$ and the identity map on $C_{0}$. Then there is a unique isomorphism $\alpha^{\prime}$ such that $\alpha^{\prime} \beta^{\prime}=\gamma^{\prime}$. We summarize in the diagram

$$
C_{0} \oplus T \stackrel{\beta^{\prime}}{\longrightarrow}\left(C_{0} \oplus T\right) /^{i} T \stackrel{a^{\prime}}{\longrightarrow} C_{0} \oplus U, \quad \gamma^{\prime}=\alpha^{\prime} \beta^{\prime} .
$$

We intend to prove the existence of a type-1 extension $\mathbb{E}$ of $\mathfrak{A}$ such that for all $R\left[\alpha_{1}, \cdots, \alpha_{i+j}\right] \in \pi^{\boldsymbol{e}}$,

$$
\alpha^{\prime}\left(\left(R\left[\alpha_{1}, \cdots, \alpha_{i+j}\right]+{ }^{i} T\right) /{ }^{i} T\right)=R^{*}\left[\alpha_{1}, \cdots, \alpha_{j}\right] \text { where } R^{*}=a\left(\left(R+{ }^{i} T\right) /{ }^{i} T\right) .
$$

It will then follow from Theorem 1.7 that $\mathbb{E}_{i} \cong$ ๆ. As a convenience to the reader, all incidence structures under consideration are displayed in Figure 1. 


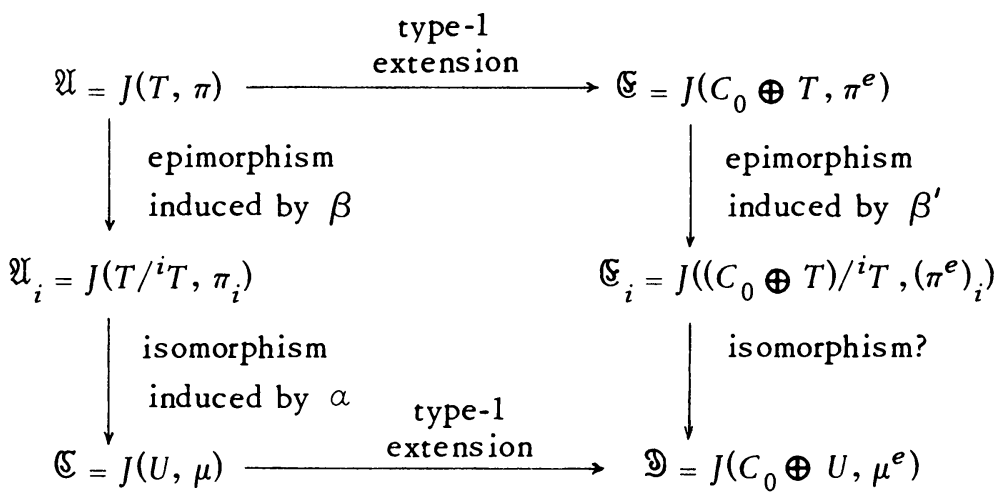

Figure 1

In the extension of $\sqrt{ }$ to $\mathscr{S}$, one must first form a subset $\nu$ of $\mu$ consisting of one line from each neighbor class of lines in $\mu$. (Note that this $\nu$ corresponds to the $\rho$ of $\$ 3$.) For each $R^{*} \in \nu$, arbitrarily select a single element $R \in \pi$ such that $\gamma(R)=R^{*}$. Denote the set of all such $R$ by $\rho$. Corollary 2.8 implies that $\rho$ consists of exactly one line from each neighbor class of lines in $\pi$. Then $\rho$ is an admissible subset of $\pi$ with which to begin a type-1 extension of 2 . Corollary 2.8 also assures the possibility of labeling the lines of $\pi$ so that

$$
\alpha\left(\left(R\left(\alpha_{1}, \ldots, \alpha_{i+j-1}\right)+{ }^{i} T\right) /{ }^{i} T\right)=R^{*}\left(\alpha_{1}, \ldots, \alpha_{j-1}\right)
$$

for all lines of $\pi$.

The next step in making the extension of $\mathcal{Q}$ is to select a congruence for $C_{0}$. We take the same congruence $\pi^{\prime}$ which produces the extension of $\sqrt{5}$ to $D$. We use the one-to-one correspondence $\nu \leftrightarrow \pi^{\theta}, R^{*} \leftrightarrow R^{\prime}$ to establish the one-to-one correspondence $\rho \leftrightarrow \pi^{\prime}, R \leftrightarrow R^{\prime}$. For each $R \in \rho$, we set $R^{e}=T+R^{\prime}$ and $R^{\epsilon}=$ $U+R^{\prime}$.

From the extension of $\left(\mathcal{S}\right.$ to $\mathcal{I}$, we have (for each $R^{\epsilon}$ ) a congruence of $R^{\epsilon} /\left({ }^{j-1} U+R^{*}\right)$, one of whose components is $U /\left({ }^{j-1} U+R^{*}\right)$; the remaining components are denoted by $R^{*}[\alpha] /\left({ }^{-1} U+R^{*}\right), 0 \leq \alpha<r$. Using Corollary 2.7, we see that

$$
\left.\left(\gamma^{\prime}\right)^{-1}\left({ }^{j-1} U+R^{*}\right)=\gamma^{-1}(j-1) U+R^{*}\right)=\beta^{-1}\left(\left({ }^{i+j-1} T+R\right) /{ }^{i} T\right)={ }^{i+j-1} T+R .
$$

Since $\gamma^{\prime}\left(R^{e}\right)=R^{\epsilon}$, we see that $\gamma^{\prime}$ induces an isomorphism $\gamma_{R^{\prime}}^{\prime} R^{e} /\left({ }^{i+j-1} T+R\right)$ $\stackrel{\text { isom }}{\longrightarrow} R^{\epsilon} /\left({ }^{j-1} U+R^{*}\right)$.

Since $\left(\gamma^{\prime}\right)^{-1}\left(R^{\epsilon}\right)=R^{e},\left(\gamma^{\prime}\right)^{-1}\left(R^{*}[\alpha]\right) \subset R^{e}$ for $0 \leq \alpha<r$. We denote $\left(\gamma^{\prime}\right)^{-1}\left(R^{*}[\alpha]\right)$ by $R[\alpha]$. Then the isomorphism $\gamma_{R}^{\prime}$ assures that $R^{e} /\left({ }^{i+j-1} T+R\right)$ has a congruence with components $T /\left({ }^{i+j-1} T+R\right)$ and $R[\alpha] /\left({ }^{i+j-1} T+R\right), 0 \leq$ $a<r$. Since $\gamma^{\prime}$ is onto, the definition of $R[\alpha]$ assures that $\alpha^{\prime}\left(\left(R[\alpha]+{ }^{i} T\right) /{ }^{i} T\right)=$ $R^{*}[\alpha]$. 
Let $1 \leq l<j$. Assume we have obtained subgroups $R\left[a_{1}, \ldots, a_{m}\right]$ of $C_{0} \oplus T$ for all $R \in \rho, 0 \leq \alpha_{k}<r, 1 \leq k \leq m, m \leq l$. Assume also that

$$
R\left[\alpha_{1}, \cdots, \alpha_{m}\right]=\left(\gamma^{\prime}\right)^{-1}\left(R^{*}\left[\alpha_{1}, \cdots, \alpha_{m}\right]\right) .
$$

Here the $R^{*}\left[a_{1}, \ldots, a_{m}\right]$ are the subgroups of $C_{0} \oplus U$ chosen in the process of extending $\left(5\right.$ to $\Im$. In particular, if $\alpha_{k}=0$ for $k>l$,

$$
R^{*}\left[\alpha_{1}, \ldots, \alpha_{1}\right] /\left(R^{*}\left(\alpha_{1}, \ldots, \alpha_{j-1}\right)+{ }^{j-l-1} U\right)
$$

has a congruence whose components are

$$
\left(R^{*}\left(\alpha_{1}, \ldots, \alpha_{j-1}\right)+{ }^{j-l} U\right) /\left(R^{*}\left(\alpha_{1}, \ldots, \alpha_{j-1}\right)+{ }^{j-l-1} U\right)
$$

and

$$
R^{*}\left[\alpha_{1}, \ldots, \alpha_{l}, \beta\right] /\left(R^{*}\left(\alpha_{1}, \ldots, \alpha_{j-1}\right)+{ }^{j-l-1} U\right), \quad 0 \leq \beta<r .
$$

We denote $\left(\gamma^{\prime}\right)^{-1}\left(R^{*}\left[\alpha_{1}, \ldots, \alpha_{l+1}\right]\right)$ by $R\left[\alpha_{1}, \ldots, \alpha_{l+1}\right]$. We have

$$
\left(\gamma^{\prime}\right)^{-1}\left(R^{*}\left(\alpha_{1}, \ldots, \alpha_{j-1}\right)+{ }^{j-l-1} U\right)=\gamma^{-1}\left(R^{*}\left(\alpha_{1}, \ldots, \alpha_{j-1}\right)+{ }^{j-l-1} J\right) .
$$

Since $\alpha$ is an isomorphism, (6.2) implies the above group is

$$
\left.\beta^{-1}\left(\left(R\left(\alpha_{1}, \ldots, \alpha_{i+j-1}\right)+{ }^{i} T\right) /{ }^{i} T\right)+\gamma^{-1(j-l-1} U\right) .
$$

By Corollary 2.7, $\gamma^{-1}\left({ }^{j-l-1} U\right)={ }^{i+j-l-1} T$. Then

$$
\left(\gamma^{\prime}\right)^{-1}\left(R^{*}\left(\alpha_{1}, \ldots, \alpha_{j-1}\right)+{ }^{j-l-1} U\right)=R\left(\alpha_{1}, \ldots, \alpha_{i+j-1}\right)+{ }^{i+j-l-1} T .
$$

By (6.3), $\gamma^{\prime}\left(R\left[\alpha_{1}, \ldots, \alpha_{l}\right]\right)=R^{*}\left[\alpha_{1}, \ldots, \alpha_{l}\right]$. Then $\gamma^{\prime}$ induces an isomorphism

$$
\begin{aligned}
\gamma^{\prime \prime}: R\left[\alpha_{1}, \ldots, \alpha_{l}\right] /\left(R\left(\alpha_{1}, \ldots, \alpha_{i+j-1}\right)+{ }^{l+j-l-1} T\right) & \\
& \stackrel{\text { isom }}{\longrightarrow} R^{*}\left[\alpha_{1}, \ldots, \alpha_{l}\right] /\left(R^{*}\left(\alpha_{1}, \ldots, \alpha_{j-1}\right)+{ }^{j-l-1} U\right) .
\end{aligned}
$$

We denote $\left(\gamma^{\prime}\right)^{-1}\left(R^{*}\left[\alpha_{1}, \ldots, \alpha_{l+1}\right]\right)$ by $R\left[\alpha_{1}, \cdots, \alpha_{q+1}\right]$. Since $\left(\gamma^{\prime}\right)^{-1}\left(R^{*}\left[\alpha_{1}, \cdots, \alpha_{l}\right]\right)$ $=R\left[\alpha_{1}, \ldots, \alpha_{l}\right], R\left[\alpha_{1}, \ldots, \alpha_{l}, \beta\right] \subset R\left[\alpha_{1}, \ldots, \alpha_{l}\right]$ for $0 \leq \beta<r$. Let $H$ denote $R\left(\alpha_{1}, \cdots, \alpha_{i+j-1}\right)+{ }^{i+j-l-1} T$. The isomorphism $\gamma^{\prime \prime}$ assures the existence of a congruence for $R\left[\alpha_{1}, \ldots, \alpha_{l}\right] / H$ whose components are

$$
\left(R\left(\alpha_{1}, \ldots, \alpha_{i+j-1}\right)+{ }^{i+j-l} T\right) / H \quad \text { and } \quad R\left[\alpha_{1}, \ldots, \alpha_{l+1}\right] / H, \quad 0 \leq \alpha_{l+1}<r .
$$

To see that the first component above is correct, one must again rely on (6.2) and Corollary 2.7. Proceeding in this fashion for $j-1$ steps, we obtain subgroups $R\left[\alpha_{1}, \cdots, \alpha_{j}\right]$ of $C_{0} \oplus T$ such that

$$
\alpha^{\prime}\left(\left(R\left[\alpha_{1}, \ldots, \alpha_{j}\right]+{ }^{i} T\right) /{ }^{i} T\right)=R^{*}\left[\alpha_{1}, \ldots, \alpha_{j}\right] .
$$

One may now continue (making any choices for congruences permitted by the 
type- 1 construction) until groups $R\left[\alpha_{1}, \ldots, \alpha_{i+j}\right]$ are obtained. Then

$$
\begin{aligned}
\alpha^{\prime}\left(\left(R\left[\alpha_{1}, \cdots, \alpha_{i+j}\right]+{ }^{i} T\right) /{ }^{i} T\right) & \subset \alpha^{\prime}\left(\left(R\left[\alpha_{1}, \cdots, \alpha_{j}\right]+{ }^{i} T\right) /{ }^{i} T\right) \\
& =R^{*}\left[\alpha_{1}, \cdots, \alpha_{j}\right] .
\end{aligned}
$$

By Lemma 3.2,o $\left(R\left[\alpha_{1}, \cdots, \alpha_{i+j}\right]\right)=r \cdot o(R)$. By (5.4) and Proposition 1.1(4), $o\left(R\left[\alpha_{1}, \cdots, \alpha_{i+j}\right] \cap{ }^{i} T\right)=o\left(R \cap{ }^{i} T\right)$. Then $o\left(\left(R\left[\alpha_{1}, \cdots, \alpha_{i+j}\right]+{ }^{i} T\right) /{ }^{i} T\right)=$ $r \cdot d\left(\left(R+{ }^{i} T\right) /{ }^{i} T\right)=r \cdot o\left(R^{*}\right)$ which, by Lemma 3.2, is equal to o $\left(R^{*}\left[\alpha_{1}, \cdots, \alpha_{j}\right]\right)$. The truth of (6.1) now follows from (6.4). Then $\mathbb{F}_{i} \cong \Im$. This completes the induction and hence, the proof of the theorem.

Corollary 6.4. For $1 \leq j \leq i$, let $\mathfrak{Q}^{j}$ be a borizontal strongly $\left(b_{j}\right)$-uniform translation H-plane. Assume every $\mathfrak{B}^{j}$ bas the same invariant $r$. Let $n(j)$ denote $\sum_{l=1}^{j} b_{l}$, and set $m(j)=n(j)-b_{j}, b=n(i)$. There exists a borizontal strongly $b$ uniform translation H-plane \& with invariant $r$ such that $\mathbb{Q}^{j} \cong\left({ }^{n(j)} \mathfrak{U}_{m(j)}\right.$ for every $j \leq i$.

Proof. We give a proof by induction on $i$. By the induction assumption, there exists a strongly $c$-uniform translation $H$-plane $(5$ with invariant $r$ and width $c=b-b_{i}$ such that for every $j<i, \mathfrak{B}^{j} \cong\left({ }^{n(j)(S)}\right)_{m(j)}$. By Theorem 6.3 , there exists a horizontal strongly $b$-uniform translation $H$-plane of with invariant $r$ such that

$$
\mathfrak{B}^{i} \cong \mathfrak{U}_{c}=\left({ }^{n(i)} \mathfrak{U}\right)_{m(i)} \text { and } \mathfrak{S} \cong c \mathfrak{U}={ }^{n(i-1)} \mathfrak{U}
$$

Then if $j<i$,

$$
\mathfrak{B}^{j} \cong\left({ }^{n(j)(S)}\right)_{m(j)} \cong\left({ }^{n(j)(c \mathfrak{Q}))_{m(j)}=\left({ }^{n(j) \mathfrak{Q})}\right.}{ }_{m(j)} .\right.
$$

The equality of $n(j)\left({ }^{c} \mathcal{U}\right)$ and $n(j)$ U follows from Lemma 1.2 together with the observation that $\mathcal{U}$ and $c \mathcal{U}$ both induce the same parallel relation into ${ }^{n(j)} 0$.

As a special case of Corollary 6.4, we have

Remark 6.5. For $1 \leq j \leq b$, let $\mathbb{R}^{j}$ be a customary translation plane of order $r$. Then there exists a horizontal strongly $b$-uniform translation $H$-plane Of with invariant $r$ such that $\mathscr{U}(j) \cong \mathfrak{R}^{j}$ for $1 \leq j \leq b$.

\section{REFERENCES}

1. J. André, Über nicht-Desarguessche Ebenen mit transitiver Translationsgruppe, Math. Z. 60 (1954), 156-186. MR 16, 64.

2. B. Artmann, Hjelmslev-Ebenen mit verfeinerten Nachbarschafts-relationen, Math. Z. 112 (1969), 163-180. MR 40\#3420.

3. P. Dembowski, Finite geomertries, Ergebnisse der Mathematik und ihrer Grenzgebiete, Band 44, Springer-Verlag, Berlin and New York, 1968. MR 38 \#1597.

4. D. A. Drake, On n-uniform Hjelmslev planes, J. Combinatorial Theory 9 (1970), 267-288. MR $42 \# 3667$. 
5. D. A. Drake, Coordinization of H-planes by H-modules, Math. Z. 115 (1970), 79-103. MR $41 \# 7524$.

6. - The translation groups of n-uniform translation Hjelmslev planes, Pacific J. Math. 38 (1971), 365-375.

7. E. Kleinfeld, Finite Hjelmslev planes, Illinois J. Math. 3 (1959), 403-407. MR 21 \#5934.

8. W. Klingenberg, Desarguessche Ebenen mit Nachbarelementen, Abh. Math. Sem. Univ. Hamburg 20 (1955), 97-111. MR 17, 522.

9. H. Lüneburg, Affine Hjelmslev-Ebenen mit transitiver Translationsgruppe, Math. Z. 79 (1962), 260-288. MR $25 \# 1479$.

10. P. Y. Bacon, Strongly n-uniform and level n Hjelmslev planes, Math. Z. 127 (1972), 1-9.

DEPARTMENT OF MATHEMATICS, UNIVERSITY OF FLORIDA, GAINESVILLE, FLORIDA 32603 